\title{
Electronic Structure and Magnetic Interactions in the Radical Salt $[\mathrm{BEDT}-\mathrm{TTF}]_{2}\left[\mathrm{CuCl}_{4}\right]$
}

\author{
Carmen J. Calzado, Bárbara Rodríguez-García, José Ramón Galán Mascarós \\ and Norge Cruz-Hernández
}

\author{
†Departamento de Química Física, Universidad de Sevilla, c/Profesor García Gonzalez, s/n, E-41012, Sevilla, Spain \\ ${ }^{\ddagger}$ Institute of Chemical Research of Catalonia (ICIQ), The Barcelona Institute of Science and Technology (BIST), Av. \\ Països \\ Catalans, 16, Tarragona, E-43007, Spain \\ $\S$ ICREA, Pg. Lluís Companys, 23, E-08010, Barcelona, Spain \\ "Departamento de Física Aplicada I, Escuela Politecnica Superior, Universidad de Sevilla, Sevilla, E-41011, Spain
}

\begin{abstract}
The magnetic behavior and electric properties of the hybrid radical salt $[\mathrm{BEDT}-\mathrm{TTF}]_{2}\left[\mathrm{CuCl}_{4}\right]$ have been revisited through extended experimental analyses and DDCI and periodic DFT plane waves calculations. Single crystal X-ray diffraction data have been collected at different temperatures, discovering a phase transition occurring in the 250-300 $\mathrm{K}$ range. The calculations indicate the presence of intradimer, interdimer, and organic-inorganic $\pi-d$ interactions in the crystal, a magnetic pattern much more complex than the Bleaney-Bowers model initially assigned to this material. Although this simple model was good enough to reproduce the magnetic susceptibility data, our calculations demonstrate that the actual magnetic structure is significantly more intricate, with alternating antiferromagnetic 1D chains of the organic BEDT-TTF${ }^{+}$radical, connected through weak antiferromagnetic interactions with the $\mathrm{CuCl}_{4}{ }^{2-}$ ions Combination of experiment and theory allowed us to unambiguously determine and quantify the leading magnetic interactions in the system. The density-of-states curves confirm the semiconductor nature of the system and the dominant organic contribution of the valence and conduction band edges. This general and combined approach appears to be fundamental in order to properly understand the magnetic structure of these complex materials, where experimental data can actually be fitted from a variety of models and parameters.
\end{abstract}

\section{INTRODUCTION}

Tetrathiafulvalene (TTF) and its derivatives form a remarkable family of crystalline organic conductors. The combination of TTF with electroactive anions has resulted in a large class of hybrid materials showing multifunctionality as magnetic organic metals ${ }^{1}$ and superconductors, ${ }^{2}$ ferromagnetic metals, ${ }^{3}$ chiral conductors, ${ }^{4}$ or switchable conductors. ${ }^{5,6}$ Among the anions, tris(oxalato)metalate complexes ${ }^{6}$ and metal halides have been employed to introduce magnetic properties in these hybrid materials, and in particular copper halides due to their large versatility, both structural and electronic, with multiple oxidation states.

Recently, the chemical synthesis of [BEDT-TTF $]_{2}\left[\mathrm{CuCl}_{4}\right]$ (1) was reported, ${ }^{7}$ (BEDT-TTF $=$ bis(ethylenedithio)tetrathiafulvalene), resulting from the chemical oxidation of BEDT-TTF by the $\left[\mathrm{Cu}_{4} \mathrm{OCl}_{10}\right]^{4-}$ anion. This is an unusual procedure, since most of the TTF-derivatives hybrid salts have been obtained by selective oxidation of the neutral species during electrocrystallization, such is the case of the salts $[\mathrm{BEDT}-\mathrm{TTF}]_{3}\left[\mathrm{CuBr}_{3}\right],{ }^{8}[\mathrm{BEDT}-\mathrm{TTF}]_{2}\left[\mathrm{FeX}_{4}\right], \mathrm{X}=\mathrm{Cl}, \mathrm{Br}{ }^{9}$ and $[\mathrm{BEDT}-\mathrm{TTF}]_{2}\left[\mathrm{MCl}_{4}\right], \mathrm{M}=\mathrm{Zn},{ }^{10} \mathrm{Au} .{ }^{11}$ The crystal structure of 1 was reported at $100 \mathrm{~K}$. ${ }^{7}$ This salt crystallizes in the triclinic PГspace group, with two independent BEDT-TTF
(Figure 1). The anion presents a Jahn-Teller distortion, with a geometry in between square planar and tetrahedral. The organic molecules form 1D stacks along the a axis. The BEDTTTF units are dimerized, with a regular $A B A B$ pattern; the $S \cdots S$ contacts are above $3.4 \AA$ in the $\mathrm{AB}$ dimers and above $3.8 \AA$ in the interdimer BA contacts (Figure 2). Each BEDT-TTF molecule carries out one positive charge; that is, the organic compound is a cationic radical, BEDT-TTF ${ }^{+}$. The $\mathrm{CuCl}_{4}{ }^{2-}$ anions are also arranged forming columns along the a axis (with $\mathrm{Cu} \cdots \mathrm{Cu}$ distances of $8.8 \AA$ ), interacting with the adjacent BEDT-TTF units, with short $\mathrm{Cl} \cdots \mathrm{S}$ contacts (between 3.2 and $3.5 \AA$ ). The compound was characterized as a semiconductor with very low room temperature electron delocalization.

The EPR spectra show only one signal with small temperature dependence that was assigned to the metal centers. This absence of any signal from the organic network supported the presence of strong intradimer antiferromagnetic couplings. The thermal dependence of the $X_{m} \top$ product (with $X_{m}$ being the molar magnetic susceptibility) was reproduced with a spin model containing isolated $\mathrm{AB}$ dimers composed of two BEDT-TTF ${ }^{+}$radical units (Bleaney-Bowers model), plus 


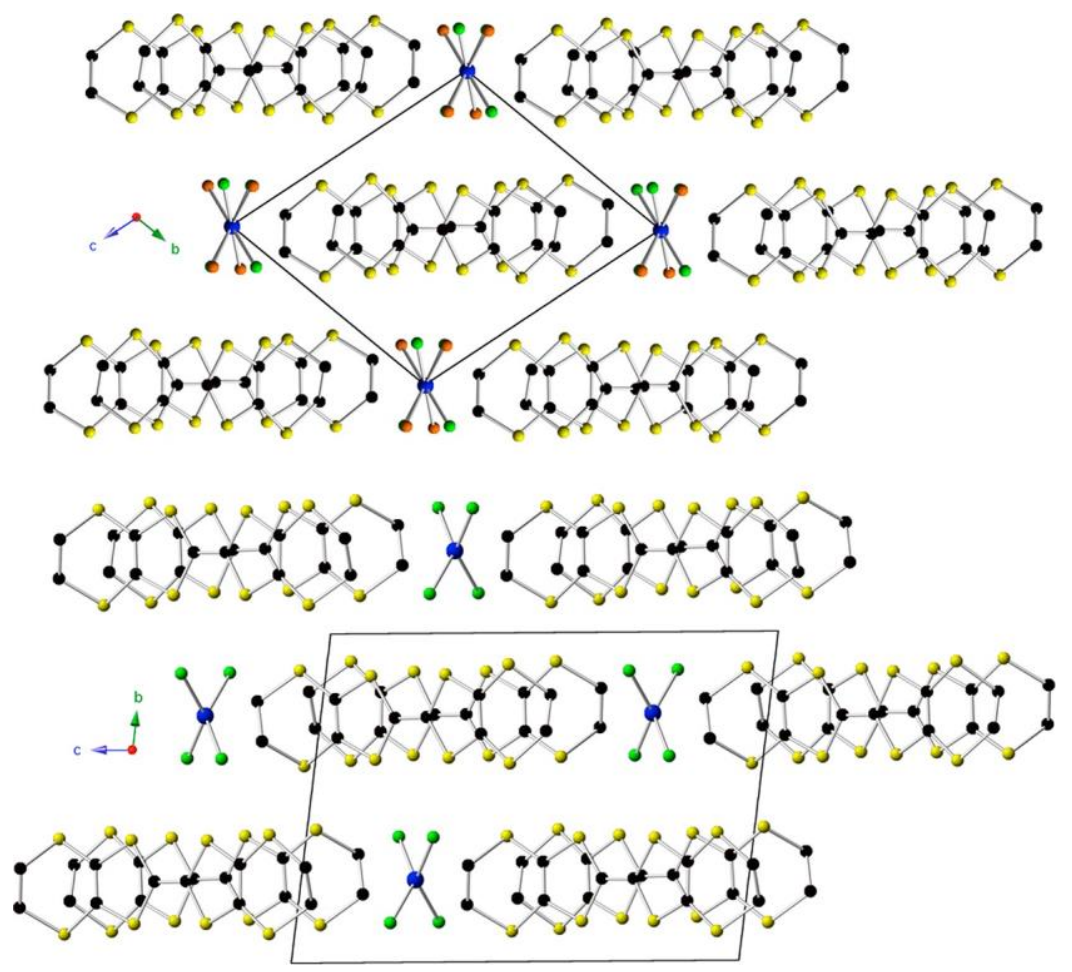

Figure 1. Projection of the crystal structure for BEDT- $\mathrm{TTF}_{2}\left[\mathrm{CuCl}_{4}\right]$ on the bc plane at $300 \mathrm{~K}$ (top) and $100 \mathrm{~K}$ (bottom). The disorder in the $\mathrm{CuCl}_{4}{ }^{2-}$ anions is represented with one orientation in green and the other in orange.
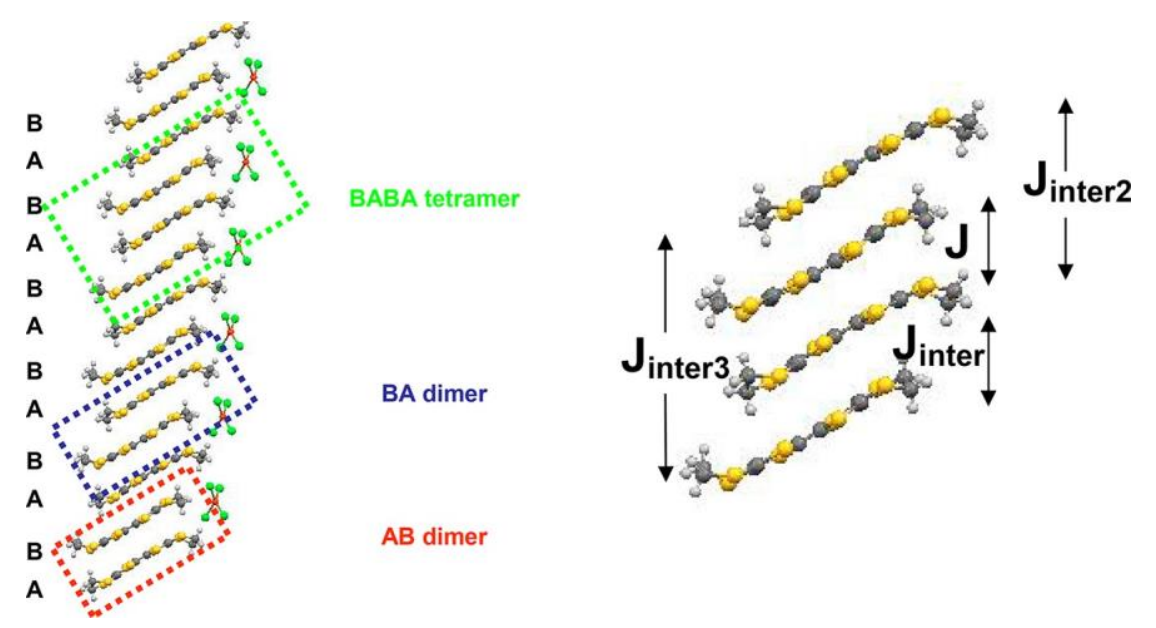

Figure 2. (left) The $\mathrm{AB}, \mathrm{BA}$, and BABA clusters employed to evaluate the magnetic coupling along the BEDT-TTF stacks. The $\mathrm{CuCl}_{4}{ }^{2-}$ anion has not been included in any of these models. (right) Different magnetic interactions along the BEDT-TTF stack.

the paramagnetic contribution coming from the isolated $\mathrm{CuCl}_{4}{ }^{2-}$ anions. The best fit corresponded to an intradimer coupling constant $\mathrm{J}=-432 \mathrm{~cm}^{-1}$ for the isotropic Heisenberg Hamiltonian $\mathrm{H}=-J \mathrm{~S}_{1} \mathrm{~S}_{2}$. Although this simplified model was able to fit the experimental data, a closer look at the crystal structure reported opens some questions to such simple magnetostructural correlations. Interdimer distances are comparable to the corresponding intradimer ones, suggesting a much more complex magnetic structure. Indeed, the BEDTTTF packing cannot warrant the complete absence of interdimer interactions and/or additional anion-cation interactions.

It is the aim of this work to inspect in depth the origin of the magnetic behavior and electric properties of this compound through extended experimental and computational analyses. To this end, single crystal X-ray diffraction data have been obtained at different temperatures. The resulting structures are used to build correct computational models where the intradimer, interdimer, and anion-cation interactions have been evaluated by means of Difference Dedicated Configuration Interaction (DDCI) calculations. The study is completed with complementary plane waves periodic calculations that provide information about the density of states and transport properties of the system. Our results indicate that the macroscopic behavior of the crystal comes from the tuning of the whole set of interactions, intradimer and interdimer in the organic component and the organic-inorganic contributions. Remarkably, the intradimer interaction is so-strong with respect to $k_{B} T$ that the corresponding singlet remains exclusively populated in the working temperatures. Thus, the observed changes in the 
Table 1. Structural Parameters for 1 at Different Temperatures

\begin{tabular}{|c|c|c|c|c|c|}
\hline & $100 \mathrm{~K}$ & $150 \mathrm{~K}$ & $200 \mathrm{~K}$ & $250 \mathrm{~K}$ & $300 \mathrm{~K}$ \\
\hline Space group & $\mathrm{P} \bar{I}^{-}$ & $\mathrm{P} \overline{1}$ & $\mathrm{P} \overline{1}$ & $\mathrm{P} \overline{1}$ & $\mathrm{P} \overline{1}$ \\
\hline a $(\AA)$ & $8.7978(10)$ & $8.8247(6)$ & $8.8586(8)$ & $8.8513(7)$ & $8.8636(8)$ \\
\hline b $(\AA)$ & $12.193(2)$ & $12.2143(8)$ & $12.2524(13)$ & $12.2433(9)$ & $9.5408(8)$ \\
\hline$c(\AA)$ & $15.767(2)$ & $15.7731(10)$ & $15.830(2)$ & $15.8259(11)$ & $10.4744(9)$ \\
\hline$\alpha(\operatorname{deg})$ & $95.33343(5)$ & $95.369(2)$ & $95.459(4)$ & $95.448(2)$ & $104.578(2)$ \\
\hline$\beta$ (deg) & $95.042(6)$ & $94.921(2)$ & $95.755(3)$ & $94.432(2)$ & $96.982(3)$ \\
\hline Y (deg) & $107.352(5)$ & $107.460(2)$ & $107.442(4)$ & $107.428(2)$ & $104.423(2)$ \\
\hline$V\left(\AA^{3}\right)$ & $1595.4(4)$ & $1603.2(2)$ & $1620.4(3)$ & $1618.8(2)$ & $814.04(12)$ \\
\hline Z & 2 & 2 & 2 & 2 & 1 \\
\hline
\end{tabular}

Table 2. Distances and Angles

\begin{tabular}{|c|c|c|c|c|c|}
\hline & $100 \mathrm{~K}$ & $150 \mathrm{~K}$ & $200 \mathrm{~K}$ & $250 \mathrm{~K}$ & $300 \mathrm{~K}^{\mathrm{a}}$ \\
\hline \multicolumn{6}{|c|}{ Distances $(\AA)$} \\
\hline $\mathrm{Cu}-\mathrm{Cl} 1$ & $2.2639(8)$ & $2.2644(8)$ & $2.2733(8)$ & $2.2548(11)$ & $2.159(3)$ \\
\hline $\mathrm{Cu}-\mathrm{Cl} 2$ & $2.2550(8)$ & $2.2546(8)$ & $2.2624(8)$ & $2.2629(12)$ & $2.204(2)$ \\
\hline $\mathrm{Cu}-\mathrm{Cl} 3$ & $2.2382(9)$ & $2.2404(9)$ & $2.2459(9)$ & $2.2376(12)$ & $2.285(2)$ \\
\hline $\mathrm{Cu}-\mathrm{Cl} 4$ & $2.2529(8)$ & $2.2551(8)$ & $2.2652(8)$ & $2.2533(12)$ & $2.385(2)$ \\
\hline $\mathrm{Cu}-\mathrm{Cl}_{\text {Avg. }}$ & 2.253 & 2.254 & 2.262 & 2.252 & 2.258 \\
\hline \multicolumn{6}{|c|}{ Angles (deg) } \\
\hline $\mathrm{Cl} 1-\mathrm{Cu}-\mathrm{Cl} 2$ & $96.29(3)$ & $96.30(3)$ & $96.27(3)$ & $95.98(4)$ & $95.87(9)$ \\
\hline $\mathrm{Cl} 1-\mathrm{Cu}-\mathrm{Cl} 3$ & $142.44(4)$ & $142.66(4)$ & $142.65(4)$ & $143.87(6)$ & $145.07(9)$ \\
\hline $\mathrm{Cl} 1-\mathrm{Cu}-\mathrm{Cl} 4$ & $96.74(3)$ & $96.64(3)$ & $96.76(3)$ & $96.35(4)$ & 93.94(9) \\
\hline $\mathrm{Cl} 2-\mathrm{Cu}-\mathrm{Cl} 3$ & $96.16(3)$ & $96.06(3)$ & $96.05(3)$ & $95.65(4)$ & 93.94(9) \\
\hline $\mathrm{Cl} 2-\mathrm{Cu}-\mathrm{Cl} 4$ & $141.93(3)$ & $142.29(3)$ & $142.30(3)$ & $143.33(5)$ & $145.07(9)$ \\
\hline $\mathrm{Cl} 3-\mathrm{Cu}-\mathrm{Cl} 4$ & $94.90(3)$ & $94.73(3)$ & $94.67(3)$ & $94.40(5)$ & $95.87(9)$ \\
\hline
\end{tabular}

magnetic susceptibility are due to the perturbation created by the interdimer interactions, and not intradimer ones. Actually, the best fit $J$ value resulting from the Bleaney-Bowers model represents just an ef fective coupling, whose origin is not in the intradimer but in the interdimer interactions.

\section{CRYSTAL STRUCTURE AT DIFFERENT TEMPERATURES}

We collected multiple temperature single-crystal X-ray diffraction data in the 100-300 $\mathrm{K}$ range (Table 1). At room temperature, the triclinic unit cell contains two BEDT-TTF molecules and $\mathrm{a} \mathrm{CuCl}_{4}{ }^{2-}$ anion. The BEDT-TTF molecules stack along the a axis (Figure 1) forming 1D chains, following a regular $\mathrm{ABAB}$ pattern. These chains are strongly dimerized, with multiple very short intradimer $S \cdots S$ contacts between the core TTF sulfur atoms (shortest $\mathrm{S} \cdots \mathrm{S}$ contacts $=3.438(9)$ and $3.510(9) \AA)$. The interdimer shortest contacts are above $3.8 \AA$ along the chains. However, there are also close contacts between chains, with a minimum $3.45(1) \AA \mathrm{S} \cdots \mathrm{S}$ distance.

These contacts are mediated by cation-anion interactions, directed by weak hydrogen bonds to the methylene groups $(\mathrm{C} \cdots \mathrm{Cl}$ distance $=2.75(1) \AA)$. The $\mathrm{CuCl}_{4}{ }^{2-}$ moiety shows the expected distorted pseudo-4-fold symmetry, in between square planar and tetrahedral (Figure 1). The average $\mathrm{Cu}-\mathrm{Cl}$ distance is $2.26 \AA$, and $\mathrm{Cl}-\mathrm{Cu}-\mathrm{Cl}$ angles are $\approx 93^{\circ}$ for adjacent and $\approx 145^{\circ}$ for opposite chlorides (Table 2 ). In the crystal structure, the $\mathrm{CuCl}_{4}{ }^{2-}$ moieties adopt two orthogonal orientations (Figure 1), with identical probability (50:50). This structural disorder generates a crystallographic center of symmetry at the
$\mathrm{Cu}$ position, although the $\mathrm{Cu}$ complex is not centrosymmetric. Crystallographically, this disposition could also correspond to two orthogonal square-planar $\mathrm{Cu}$ ions (part1 and part2, hereafter). Although this is chemically incorrect (as confirmed by the low temperature data), both descriptions are plausible, each one describing in a different way the surroundings of the radical cations. Both models will be explored in our calculations.

When decreasing temperature, the anions order progressively, adopting almost exclusively one of the two orientations below $250 \mathrm{~K}$. This crystallographic order is enough (70:30) to destroy the center of symmetry. The absence of the center of symmetry doubles the unit cell due to this localization (Figure 1b). Down $200 \mathrm{~K}$, the unit cell remains essentially constant, and additional shrinking is observed at lower temperatures. The change in unit cell, due to anion crystallographic ordering, does not affect the packing in the organic network, exhibiting the same pseudodimerized structure. At $100 \mathrm{~K}$ (as our low temperature limiting case), the BEDT-TTF molecules stacking along the a axis show shorter intradimer $S \cdots S$ contacts between core TTF sulfur atoms (shortest $S \cdots S$ contacts $=3.399(9)$, $3.440(9)$, and $3.420(9) \AA)$, indicating slightly stronger dimerization. On the contrary, the interchain contacts are

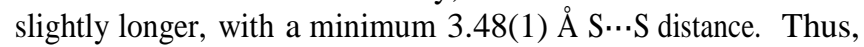
the effect of these crystallographic changes in the electronic properties of this material should be very limited.

Since below $250 \mathrm{~K}$ both the unit cell and the $\mathrm{CuCl}_{4}{ }^{2-}$ distances and angles do not show significant changes, we have 
restrained our computational models to the structures of the lower and upper accessed temperatures (100 and $300 \mathrm{~K}$ ).

\section{DESCRIPTION OF THE MODELS AND COMPUTATIONAL DETAILS}

Finite cluster and periodic calculations have been combined to analyze the electronic structure of the system and provide information about the nature and amplitude of the magnetic interactions along the BEDT-TTF chains and between the organic radicals and the $\mathrm{CuCl}_{4}{ }^{2-}$ ions.

3.1. Finite Cluster Calculations. First, the electronic structure of one isolated BEDT-TTF ${ }^{+}$molecule has been analyzed by means of a set of CASSCF calculations on the doublet ground state, employing active spaces of increasing size and the X-ray geometrical data of one of the BEDT-TTF molecules at $100 \mathrm{~K}$.

Different models have been employed to explore the magnetic pathways on this system. Using the X-ray data collected at 100 and $300 \mathrm{~K}$ without any additional refinement, we have selected two, three, and four BEDT-TTF molecules with different purposes. The AB and BA dimers (Figure 2) provide the information required to evaluate the intradimer, J, and interdimer, $J_{\text {inter }}$, interactions along the BEDT-TTF stacks. The presence of non-negligible interactions between the $\mathrm{CuCl}_{4}{ }^{2-}$ ions and the organic spin carriers has been checked with models including two $\mathrm{CuCl}_{4}{ }^{2-}$ anions, named as $\mathrm{AB}$ $+2 \mathrm{CuCl}_{4}$ and $\mathrm{BA}+2 \mathrm{CuCl}_{4}$, respectively (Figures 3 and 4 ).

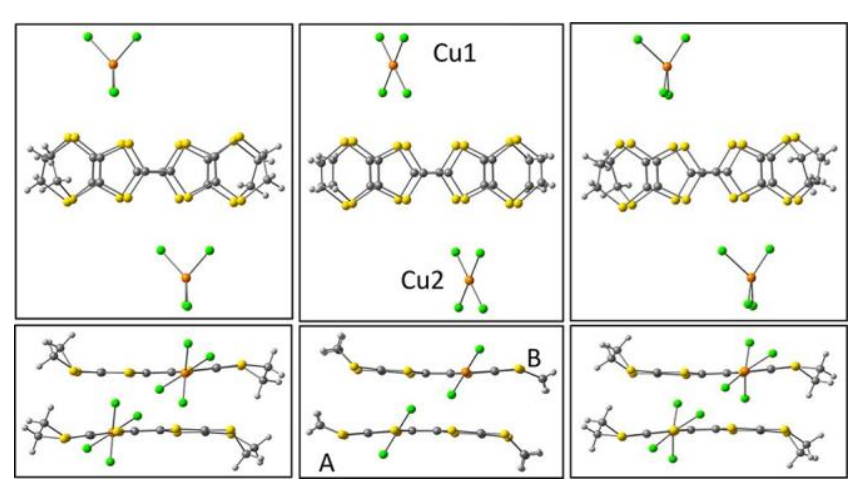

Figure 3. Top and lateral views of $\mathrm{AB}+2 \mathrm{CuCl}_{4}$ model extracted from the $\mathrm{X}$-ray data measured at $100 \mathrm{~K}$ (left), $300 \mathrm{~K}$-average (middle), and 300 K-distorted (right).

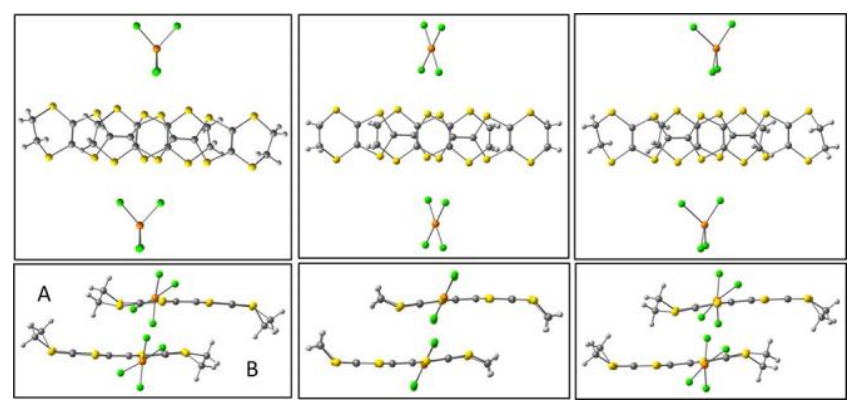

Figure 4. Top and lateral views of $\mathrm{BA}+2 \mathrm{CuCl}_{4}$ model extracted from the X-ray data measured at $100 \mathrm{~K}$ (left), $300 \mathrm{~K}$-average (middle), and $300 \mathrm{~K}$ - distorted (right).

Additionally, a model containing two organic molecules of two neighbor BEDT-TTF chains connected with a $\mathrm{CuCl}_{4}{ }^{2-}$ anion has also been considered ( $\mathrm{A}-\mathrm{Cu}-\mathrm{B}$ model, Figure 5). These

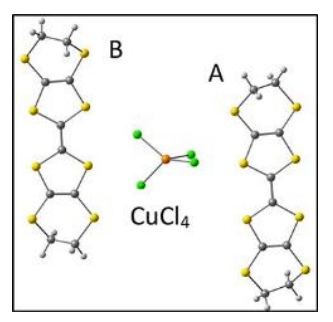

Figure 5. Top view of the $\mathrm{A}-\mathrm{Cu}-\mathrm{B}$ model at $100 \mathrm{~K}$.

five models have been directly extracted from the X-ray crystallographic data measured at 100 and $300 \mathrm{~K}$. In the case of the structure at $100 \mathrm{~K}$, the $\mathrm{CuCl}_{4}{ }^{2-}$ ions have been incorporated exclusively in their major orientation. For the $300 \mathrm{~K}$ structures, two different sets of coordinates have been employed for the $\mathrm{CuCl}_{4}{ }^{2-}$ ions, those representing one of the possible orientations of the distorted structure $(300 \mathrm{~K}$-distorted in the following) and those representing an average squareplanar structure (300 K-average). The main difference among these two sets of coordinates concerns the relative position of the $\mathrm{Cl}$ atoms with respect to the organic molecule.

As well, the trimer ABA and the tetramer BABA are used to confirm the evaluations of $J$ and $J_{\text {inter }}$ on the dimers and to detect the presence of longer-range interactions, such as the coupling between second-neighbor units, $J_{\text {inter2 }}$, or thirdneighbor units, $J_{\text {inter3 }}$ (Figure 2). These two clusters have been selected from the X-ray data at $100 \mathrm{~K}$. Notice that all the considered interactions are through-space interactions, in contrast to the through-bond interactions governing magnetism in most of the transition-metal based magnetic systems.

Spin Model. Several approaches have been employed to determine the amplitude and nature of the magnetic interaction along the BEDT-TTF stacks and between the BEDT-TTF molecules and the $\mathrm{CuCl}_{4}{ }^{2-}$ anions. In all cases it has been assumed that the magnetic interactions can be correctly represented by means of a Heisenberg-Dirac Van Vleck (HDVV) model:

$$
\hat{H}_{H D V V}=-\sum_{i<j} J_{i j}\left(\hat{S_{i} S_{j}}-\frac{1}{4} I\right)
$$

where $\hat{S}$ and $I$ are the spin and identity operators and $J_{i j}$ corresponds to the coupling constant between the spins in centers $i$ and $j$. The last term in this definition makes zero the energy of the highest spin multiplicity state. $J$ is negative for antiferromagnetic couplings and positive for ferromagnetic interactions. In the case of dimers $A B$ with two unpaired electrons on two active orbitals, the intradimer coupling can be obtained just from the energy difference between the singlet and triplet states, $J=E(S)-E(T)$. In the case of the BA unit, the singlet-triplet splitting equals the interdimer coupling constant, $J_{\text {inter. }}$.

In the trimer $\mathrm{ABA}$, with three electrons on three orbitals, it is possible to distinguish three different interactions: $J$ for the coupling between $\mathrm{A} 1$ and B1 radicals in the dimer, $J_{\text {inter }}$ for the interaction between $\mathrm{B} 1$ and $\mathrm{A} 2$, and $J_{\text {inter2 }}$ for the coupling between A1 and A2. The HDVV Hamiltonian has three solutions, the quartet and two doublet states, whose energies depend on the three parameters. If the $J_{\text {inter2 }}$ interaction is neglected, it is possible to estimate the $J$ and $J_{\text {inter }}$ constants from the energy of the three states (eq S1 in the Supporting Information file). ${ }^{12}$ To additionally estimate the $J_{\text {inter2 }}$ interaction, we need to calculate the HDVV matrix elements 
Table 3. Composition and Spin Density of the Ground State Wave Function of the BEDT-TTF ${ }^{+}$Radical

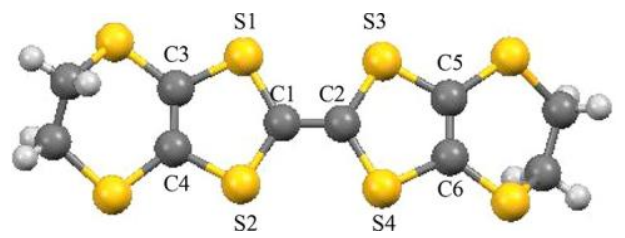

\begin{tabular}{|c|c|c|c|c|c|c|c|c|c|c|c|}
\hline \multirow[b]{2}{*}{$\mathrm{CAS}(\mathrm{MO} / \mathrm{e})$} & \multirow[b]{2}{*}{$\% \mathrm{ROHF}$} & \multicolumn{10}{|c|}{ Spin density } \\
\hline & & $\mathrm{C} 1$ & $\mathrm{C} 2$ & $\mathrm{C} 3$ & $\mathrm{C} 4$ & $\mathrm{C} 5$ & C6 & $\mathrm{S} 1$ & $\mathrm{~S} 2$ & S3 & S4 \\
\hline$(1 / 1)$ & 100 & 0.161 & 0.147 & 0.023 & 0.025 & 0.031 & 0.030 & 0.123 & 0.145 & 0.144 & 0.145 \\
\hline$(2 / 1)$ & 100 & 0.161 & 0.147 & 0.023 & 0.025 & 0.031 & 0.030 & 0.124 & 0.144 & 0.143 & 0.145 \\
\hline$(6 / 5)$ & 92 & 0.183 & 0.165 & 0.018 & 0.021 & 0.025 & 0.023 & 0.125 & 0.143 & 0.135 & 0.144 \\
\hline$(10 / 13)$ & 88 & 0.153 & 0.120 & 0.018 & 0.020 & 0.027 & 0.024 & 0.137 & 0.160 & 0.155 & 0.161 \\
\hline
\end{tabular}

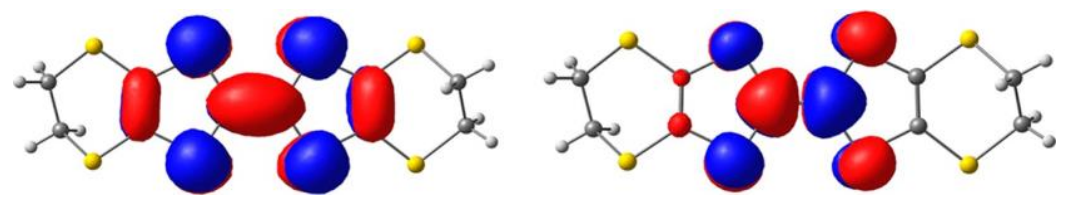

Figure 6. (left) SOMO and (right) LUMO for the BEDT-TTF ${ }^{+}$radical molecule.

(Table S1), using the energy and wave function of the three states.

In the case of $\mathrm{AB}+2 \mathrm{CuCl}_{4}, \mathrm{BA}+2 \mathrm{CuCl}_{4}$, and $\mathrm{BABA}$ models, there are four interacting $S=1 / 2$ spins in the system, and five different interactions can be distinguished. The six lowest states with $S_{z}=0$ have been calculated, and the amplitude of the interactions has been extracted from the elements of the effective HDVV matrices (Tables S2 and S3).

In all the models, the energy of the magnetic states involved in the coupling is evaluated by means of Difference Dedicated Configuration Interaction (DDCI) calculations, ${ }^{13}$ combined with the EXSCI approach ${ }^{14}$ to make feasible the calculations on the more extended models (see the SI file for a more detailed description). Details of the EXSCI approach and recent

applications can be found in refs 14 and 15-19, respectively.

The basis sets employed are described in the SI file. All CASSCF calculations have been performed using the MOLCAS@UU 8.0 program package. ${ }^{20}$ The CASDI code Th,22 $^{21}$ has been used for DDCI calculations, combined with the Lewis program ${ }^{15}$ to perform the localization of the molecular orbitals.

3.2. Periodic Calculations. The electronic and the structural properties of [BEDT-TTF $]_{2}\left[\mathrm{CuCl}_{4}\right]$ salts have also been investigated by Density Functional Theory (DFT) calculations in a periodic framework, considering spin-polarized solutions, as implemented in the Vienna Ab-initio Simulation Package (VASP). ${ }^{23}$ Full geometry optimizations, including both atom positions and lattice parameters were performed, using the optB86b-vdW approach. ${ }^{24}$ We started from triclinic cells with $P \Gamma$ symmetry group structure, and we always obtained a final structure with the same symmetry (see the SI file for further details). The cell used includes four organic molecules [BEDT-TTF] and two inorganic $\left[\mathrm{CuCl}_{4}{ }^{2-}\right]$ ions, i.e., six unpaired electrons per cell. Two different solutions were calculated in this work, according to the difference between number of electrons in up and down spin component $(\uparrow-\downarrow)$, i.e: $(\uparrow-\downarrow=2)$ and $(\uparrow-\downarrow=6)$. In the former solution, AFM hereafter, only two of the six active electrons per cell remain unpaired, while in the latter, FM solution, the six active electrons are parallel. In a second step, accurate electronic structures were obtained from single-point calculations with the B3LYP ${ }^{25}$ hybrid functional.

\section{RESULTS AND DISCUSSION}

4.1. Electronic Structure of the BEDT-TTF+ Unit. The electronic structure of the BEDT-TTF ${ }^{+}$molecules has been analyzed by means of a set of CASSCF calculations using active space with increasing number of electrons and orbitals. The aim is 2-fold: first, to determine the nature of the orbital/s carrying the unpaired electron and the charge distribution on the molecule, and second, to rationally choose the minimal active space for subsequent calculations on larger fragments. Two main aspects have been considered: (i) the composition of the doublet ground state wave function and (ii) the distribution of the spin density on the different atoms. The results obtained are summarized in Table 3.

CAS(1/1) contains one electron on the singly occupied molecular orbital (SOMO in Figure 6), which can be described as mainly resulting from the $\pi$ bonding between the $\mathrm{C} 1$ and $\mathrm{C} 2$ atoms. The $\mathrm{CAS}(2 / 1)$ contains additionally the corresponding virtual $\pi^{\star}$ combination (LUMO in Figure 6). Adding the MOs resulting from the $\pi$ and $\pi^{*}$ of the $\mathrm{C} 3-\mathrm{C} 4$ and $\mathrm{C} 5-\mathrm{C} 6$ bonds, we obtain the CAS(6/5), and with the $\pi$ lone pairs of $\mathrm{S} 1-\mathrm{S} 4$ atoms it results the CAS $(10 / 13)$. The weight of the ROHF determinant (only one singly occupied active orbital, the rest of active orbitals being doubly occupied) decreases as expected with the size of the CAS, but as shown in Table 3 , it still remains highly representative of the doublet ground state wave function. The interaction between two BEDT-TTF ${ }^{+}$units can then be safely modeled with an active space containing the SOMOs of each unit and two electrons, i.e. $\operatorname{CAS}(2 / 2)$.

4.2. Electronic Structure of the $A B$ and BA Dimers. Two main geometrical features govern the interactions in the $\mathrm{AB}$ and BA dimers: the intradimer $\mathrm{C} 1-\mathrm{C} 1^{\prime}$ and $\mathrm{C} 2-\mathrm{C} 2$ ' distances (see Table 3 for notation) and the $\mathrm{C} 2-\mathrm{C} 2^{\prime}-\mathrm{C} 1^{\prime}$ slippage angle between the two BEDT-TTF ${ }^{+}$units. A slippage angle of $90^{\circ}$ would correspond to a completely eclipsed $\pi$ dimer as some of the reported $\pi-[\mathrm{TCNE}]_{2}^{2-}$ dimers. $^{26,27}$ The intradimer distances and slippage angle are 3.364 and $3.408 \AA$ 


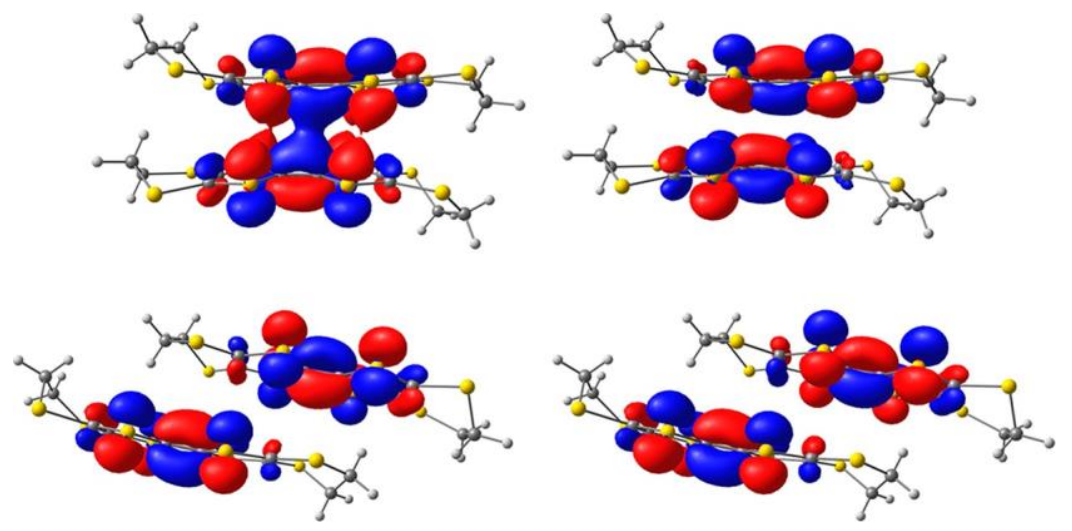

Figure 7. Bonding and antibonding combinations of the SOMOs orbitals in AB dimer (top) and BA dimer (bottom). MOs resulting from a $\mathrm{CASSCF}(2 / 2)$ calculation on the singlet state; similar shapes for those coming from the triplet state.

and $101.5^{\circ}$, respectively, in $\mathrm{AB}$, and 5.869 and $5.888 \AA$ and $139.4^{\circ}$ in dimer BA.

The electronic structure of the $\mathrm{AB}$ and BA dimers can be analyzed in a first approximation from the CASSCF wave function of the singlet ground state built on the basis of the SOMOs of each BEDT-TTF unit (a two-electron in two MOs CAS). The active orbitals are shown in Figure 7 for both systems. In the $\mathrm{AB}$ dimer, the bonding combination of the SOMOs (top left in Figure 7) presents a significant $\sigma$-overlap of the two ח-SOMOs in the intradimer area, that evokes the long multicenter bond in $\pi-[\mathrm{TCNE}]_{2}{ }^{2-}$ dimers. ${ }^{26 \mathrm{~b}, \mathrm{c}, 27}$ The occupation numbers of these MOs are 1.633 and 0.366 for the bonding and antibonding combinations, respectively. These occupation numbers indicate that the $\mathrm{AB}$ dimer cannot be described as a closed-shell singlet, but it has a non-negligible open-shell character, as confirms the valence-bond analysis of the singlet state wave function at CASSCF and DDCI levels (Table S4). The occupation numbers also indicate a large separation of the bonding and antibonding combinations, in line with the large S-T gap of about $-2500 \mathrm{~cm}^{-1}$ found at this level. In the case of BA dimer, both the longer intradimer distances and the larger slippage angle reduce the intradimer overlap, the energy separation between the bonding and antibonding MOs is smaller, the singlet presents a more enhanced open-shell character (occupations numbers are 1.210 and 0.789$)$, and the $\mathrm{S}-\mathrm{T}$ gap is significantly reduced $\left(\mathrm{J}_{\text {inter }}=\right.$ $-248 \mathrm{~cm}^{-1}$ at this level).

4.3. Evaluation of the Magnetic Coupling Constants. All the DDCI calculations have been performed with the molecular orbitals resulting from preliminary CASSCF calculations of the highest spin multiplicity solution on the basis of the minimal CAS. Hence, a CAS $(2 / 2)$ for $\mathrm{AB}$ and BA clusters with two electrons distributed on the SOMOs of the BEDT-TTF units, a CAS(3/3) for ABA fragment, and CAS(4/ 4) for $\mathrm{BABA}, \mathrm{AB}+2 \mathrm{CuCl}_{4}$, and $\mathrm{BA}+2 \mathrm{CuCl}_{4}$ models. In the two latter cases, the active space contains, together with the two BEDT-TTF SOMOs, two active orbitals centered on the $\mathrm{CuCl}_{4}^{2-}$ anions, corresponding to the antisymmetric combination of the $\mathrm{Cu} 3 \mathrm{dx}^{2}-\mathrm{y}^{2}$ orbital (in local coordinates) and the $\mathrm{Cl}$ 3 p orbitals, as shown in Figure 8 for $100 \mathrm{~K}$ and Figures S1 and S2 for $300 \mathrm{~K}$ structures.

As mentioned above, the EXSCI approach has been employed to reduce the computational cost. Table 4 contains the calculated values for $J$ and $J_{\text {inter }}$ obtained with different thresholds and computational models. The bare fragments $\mathrm{AB}$ and BA provide values of about $-3000 \mathrm{~cm}^{-1}$ and $-400 \mathrm{~cm}^{-1}$
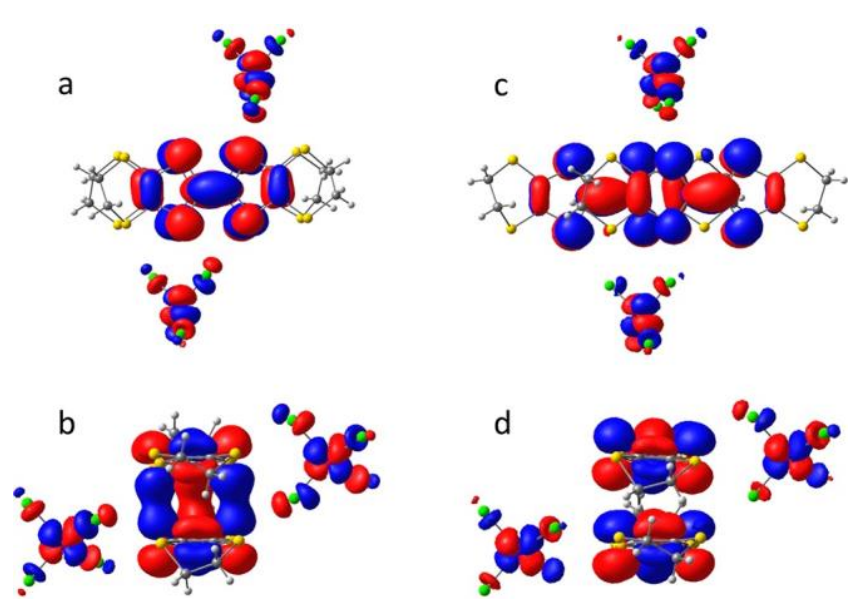

Figure 8. Top and side views of the four active orbitals for $\mathrm{AB}+2 \mathrm{CuCl}_{4}$ $(\mathrm{a}, \mathrm{b})$ and $\mathrm{BA}+2 \mathrm{CuCl}_{4}(\mathrm{c}, \mathrm{d})$ clusters at $100 \mathrm{~K}$.

for $\mathrm{J}$ and $\mathrm{J}_{\text {inter, }}$, respectively (Table 4). The EXSCI method allows a safe truncation of the CI space, involving $19 \times 10^{6}$ determinants in the case of the threshold th1, and only $6.8 \times$ $10^{6}$ determinants for threshold th2, without significant impact on the coupling values.

To check the effect of the environment on the intradimer and interdimer interactions, two additional sets of calculations have been carried out, including the two closest $\mathrm{CuCl}_{4}{ }^{2-}$ ions as point charges with values of $+1 \mathrm{e}$ and $-0.5 \mathrm{e}$ for $\mathrm{Cu}$ and $\mathrm{Cl}$ ions, respectively, or explicitly including the anions with the $\mathrm{AB}$ $+2 \mathrm{CuCl}_{4}$ and $\mathrm{BA}+2 \mathrm{CuCl}_{4}$ models. The results in Table 4 indicate a moderate effect on the intradimer interaction, while the impact is much more noticeable in the case of the interdimer coupling. Both sets of calculations provide indication of the potential modulation of these interactions by the environment, here illustrated in a qualitative way. A reliable treatment would require taking into account the electrostatic effect of the whole crystal on the AB and BA fragments, embedding the system in a set of point charges and multipoles. This approach, common in purely inorganic systems, is difficult to be implemented in this system composed by extended and highly polarizable organic ions.

In this sense, a recent study by Domingo et al. ${ }^{28}$ on the effect of the polarization of the environment on the magnetic coupling constant in neutral organic radical-based materials is remarkable. The polarization of the embedding is selfconsistently incorporated, by combining the Discrete Reaction 
Table 4. Calculated Intradimer $J$ and Interdimer $J_{\text {inter }}$ Magnetic Couplings Using Different Models and Thresholds in the DDCI Calculations $^{\mathrm{a}}$

\begin{tabular}{|c|c|c|c|c|c|c|c|c|c|}
\hline & & \multicolumn{3}{|c|}{ Bare fragments } & \multicolumn{2}{|c|}{ Point charges } & \multicolumn{2}{|c|}{ Explicit $\mathrm{CuCl}_{4}^{2-}$ ions } & \multirow{2}{*}{$\begin{array}{c}\text { Neutral dimer MOs } \\
\text { th1 }\end{array}$} \\
\hline & & th0 & th1 & th2 & th1 & th2 & th1 & th2 & \\
\hline J & $\mathrm{AB}$ & -3292 & -3041 & -2987 & -3595 & -3495 & & -2946 & -3471 \\
\hline$J_{\text {inter }}$ & BA & -411 & -420 & -370 & -333 & -297 & -154 & -147 & -193 \\
\hline
\end{tabular}

${ }^{a}$ Thresholds th1 correspond to $\mathrm{K}_{\mathrm{ij}} \geq 3 \times 10^{-4}$, th2 to $\mathrm{K}_{\mathrm{ij}} \geq 7 \times 10^{-4}$, th0 means without thresholds (i.e. all integrals, MOs and determinants are considered in CI calculations).

Field (DRF) method with the electronic structure calculations at CASPT2 and DDCI levels. The procedure requires the evaluation of the state specific induced dipoles in the embedding in a self-consistent way. ${ }^{29}$ For the inspected neutral organic radicals, the impact of the environment on the magnetic coupling was found negligible, but this result could not be transferable to ionic crystals as the [BEDT$\mathrm{TTF}]_{2}\left[\mathrm{CuCl}_{4}\right]$ salt.

It is possible to consider an alternative solution to obtain additional insights on the reliability of our estimates. In the crystal the environment compensates the two positive charges of the $\mathrm{AB}$ and $\mathrm{BA}$ fragments; then the MOs of the neutral dimers could be conceived as starting orbitals for the DDCI calculations. The same logic has been employed in a previous work regarding the magnetic coupling interactions between $\left[\mathrm{Ni}(\mathrm{dmit})_{2}\right]^{-}$radical ions. ${ }^{30}$ The starting orbitals are obtained from a CASSCF(4/4) calculation on the singlet state of the neutral dimer, where the active space contains the in-phase and out-of-phase combinations of the HOMO and LUMO of each monomer, with the same shapes as the SOMO and LUMO of the BEDT-TTF ${ }^{+}$radical (Figure 6). The subsequent DDCI calculations take into account the two positive charges of the dimer. The natural orbitals resulting from this first run are employed in an additional DDCI run to evaluate the magnetic coupling constants. The results, reported in Table 4 under the entry "Neutral dimer MOs", confirm the trends obtained with the two previous approaches.

In summary, the different strategies employed to evaluate the interdimer and intradimer coupling interactions along the BEDT-TTF stacks provide a strong antiferromagnetic coupling inside the dimer, with a $\mathrm{J}$ value of around $-3000 \mathrm{~cm}^{-1}$, and a not negligible antiferromagnetic coupling between the dimers, 1 order of magnitude smaller than $\mathrm{J}$. When compared with the reported best-fit $\mathrm{J}$ value $\mathrm{e}^{7}$ of $\mathrm{J}=-432 \mathrm{~cm}^{-1}$, it seems clear that it just corresponds to an effective coupling constant, closer to the interdimer than to the intradimer interaction.

4.4. Long-Range Interactions and Organic-Inorganic Couplings. The presence of long-range interactions has been explored by using models containing three and four BEDTTTF radicals (models ABA and BABA), while the ABA model gives information about the interaction between two equivalent BEDT-TTF units in two neighbor dimers, $J_{\text {inter2 }}$ in Figure 2. Additionally, the BABA model supplies values of the $J_{\text {inter3 }}$ interaction, which corresponds to the coupling between the two more distant BEDT-TTF units in two close neighbor dimers (Figure 2). In both models, $J_{\text {inter } 2}$ is antiferromagnetic with a value of $-43 \mathrm{~cm}^{-1}$, while $J_{\text {inter } 3}$ is null. Then, the magnetic interactions along the BEDT-TTF stacks propagate up to second neighbors, with the interaction between third-neighbor units being negligible.

We have also explored the possibility of interaction between two neighbor stacks mediated by a $\mathrm{CuCl}_{4}{ }^{2-}$ ion, with the $\mathrm{A}-$ $\mathrm{Cu}-\mathrm{B}$ model (Figure 5). In this case, the system contains three unpaired electrons, two on the organic units and a third one on the $\mathrm{CuCl}_{4}{ }^{2-}$ ion. Three different interactions can be distinguished, two between the BEDT-TTF units and the $\mathrm{CuCl}_{4}{ }^{2-}$ ion, $\mathrm{A}-\mathrm{Cu}$ and $\mathrm{B}-\mathrm{Cu}$, and a third one which represents the interstack coupling, $\mathrm{A} \cdots \mathrm{B}$. The latter is null. The hybrid organic-inorganic interactions are both antiferromagnetic in nature, with values of $-19 \mathrm{~cm}^{-1}$ for $\mathrm{A}-\mathrm{Cu}$ and -8 $\mathrm{cm}^{-1}$ for $\mathrm{B}-\mathrm{Cu}$ at $100 \mathrm{~K}$. These interactions differ on the relative orientation of the magnetic orbital localized on the $\mathrm{CuCl}_{4}{ }^{2-}$ with respect to the SOMO of the BEDT-TTF unit. The same results are obtained from the calculations on the $\mathrm{AB}$ $+2 \mathrm{CuCl}_{4}$ and $\mathrm{BA}+2 \mathrm{CuCl}_{4}$ models. In these cases, the systems contain four unpaired electrons, two on the $\mathrm{CuCl}_{4}{ }^{2-}$ ions and two on the organic units. The results are collected in Table 5

Table 5. Magnetic Coupling Constants at DDCI Level Evaluated for $\mathrm{AB}+2 \mathrm{CuCl}_{4}$ and $\mathrm{BA}+2 \mathrm{CuCl}_{4}$ Models Extracted from the Structures at 100 and $300 \mathrm{~K}$ (average and distorted) ${ }^{a}$

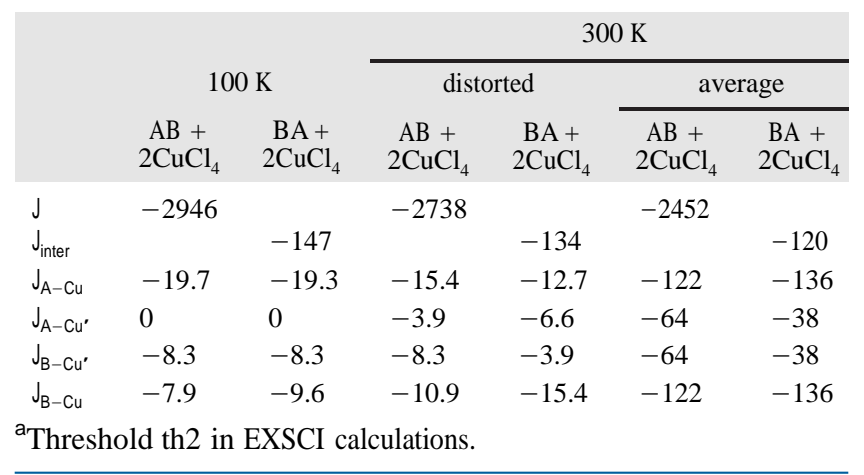

(100 K columns). They confirm the presence of weak interactions between the organic and inorganic components, of different amplitude depending on the relative orientation of the $\mathrm{Cl} 3 \mathrm{p}$ orbitals with respect to the $\pi$-SOMO orbitals. The amplitude of these through-space $\pi-d$ interactions are similar to those found between the organic radicals and the transition metal complex in molecular conductors ${ }^{31,2 \mathrm{~d}, 32}$ such as (BDA$\mathrm{TTP})_{2} \mathrm{FeCl}_{4}$ or $\lambda$-(BETS) ${ }_{2} \mathrm{FeCl}_{4}$ (BDA-TTP $=$ bis(1,3-dithian2-ylidene)tetrathiapentalene, $\mathrm{BETS}=$ bis(ethylenedithio)tetraselenafulvalene), where the admitted values for the $\pi-d$ coupling $^{33}$ are $J_{\pi d} \sim 7-10 \mathrm{~cm}^{-1}$.

4.5. Effect of Temperature. Finally, we have also performed single-point calculations on models extracted from the X-ray structure at $300 \mathrm{~K}$, with two different coordinations for the $\mathrm{Cu}$ atoms, a distorted pseudo-4-fold symmetry in the $300 \mathrm{~K}$-distorted set, and square-planar in the $300 \mathrm{~K}$-average set. The results obtained for the $\mathrm{AB}+2 \mathrm{CuCl}_{4}$ and $\mathrm{BA}+2 \mathrm{CuCl}_{4}$ models are collected in Table 5. Increasing temperature, the separation between the BEDTT-TTF ${ }^{+}$units increases, and this reduces the intradimer and interdimer couplings. The most noticeable effect is observed for the organic-inorganic $\pi-d$ 
Table 6. Equilibrium Cell Parameters for the AFM Solution Resulting from the Plane Waves Periodic Optimizations by Using the optB86b-vdW Functional

\begin{tabular}{llrrrrrr} 
& & $a(\AA)$ & $b(\AA)$ & $c(\AA)$ & $\alpha(\operatorname{deg})$ & $\beta(\operatorname{deg})$ & $\gamma(\operatorname{deg})$ \\
$100 \mathrm{~K}$ & & 8.696 & 12.173 & 15.709 & 95.53 & 94.93 & 107.30 \\
Exp. & & 8.802 & 12.187 & 15.752 & 95.33 & 95.14 & 107.42 \\
$300 \mathrm{~K}$ & part1 & 8.824 & 9.425 & 10.408 & 104.40 & 96.21 & 104.33 \\
& average & 8.918 & 9.362 & 10.280 & 103.56 & 95.51 & 105.10 \\
& distorted & 8.880 & 9.449 & 20.426 & 84.39 & 102.06 & 74.77 \\
Exp. & & 8.864 & 9.541 & 10.578 & 104.58 & 96.98 & 104.42 \\
\hline
\end{tabular}

Table 7. Molecular Package Parameters Resulting from the Plane Waves Periodic Optimizations of the AFM Solution with optB86b-vdW Functional
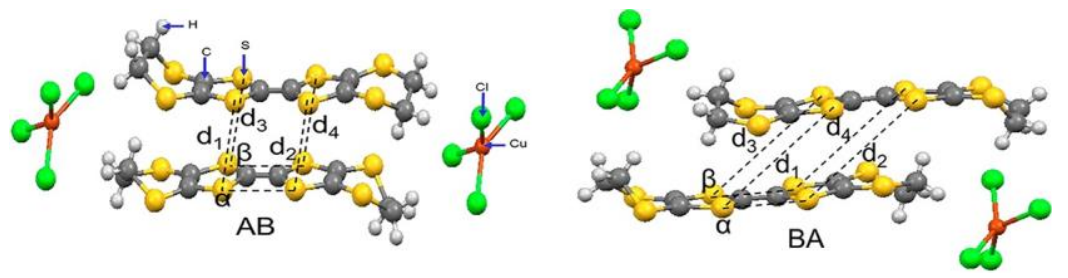

\begin{tabular}{lccccccc} 
& $\mathrm{d}_{1}(\AA)$ & $\mathrm{d}_{2}(\AA)$ & $\mathrm{d}_{3}(\AA)$ & $\mathrm{d}_{4}(\AA)$ & $\alpha(\mathrm{deg})$ & $\beta(\mathrm{deg})$ \\
& & & $100 \mathrm{~K}(\mathrm{AB} / \mathrm{BA})$ & & & \\
& $3.460 / 5.676$ & $3.474 / 5.673$ & $3.575 / 5.617$ & $3.513 / 5.660$ & $76.8 / 39.7$ & $79.5 / 41.9$ \\
Exp. & $3.390 / 5.845$ & $3.426 / 5.851$ & $3.518 / 5.832$ & $3.423 / 5.872$ & $77.2 / 39.2$ & $79.1 / 40.4$ \\
& & & $300 \mathrm{~K}(\mathrm{AB} / \mathrm{BA})$ & & & \\
part1 & $3.440 / 5.872$ & $3.492 / 5.836$ & $3.492 / 5.836$ & $3.440 / 5.872$ & $79.5 / 39.9$ & $78.6 / 40.5$ \\
average & $3.517 / 5.839$ & $3.497 / 5.872$ & $3.503 / 5.869$ & $3.506 / 5.847$ & $75.9 / 40.0$ & $76.6 / 39.8$ \\
distorted & $3.477 / 5.863$ & $3.481 / 5.845$ & $3.479 / 5.847$ & $3.474 / 5.865$ & $77.1 / 39.8$ & $76.7 / 39.9$ \\
Exp. & $3.438 / 5.889$ & $3.511 / 5.869$ & $3.511 / 5.869$ & $3.438 / 5.889$ & $78.9 / 39.4$ & $78.4 / 39.9$ \\
\hline
\end{tabular}

Table 8. Energy Expressions per Cell (relative to FM phase) for the Two Magnetic Solutions for 1 Optimized Using the optB86b-vdW Functional ${ }^{\mathrm{a}}$

\begin{tabular}{|c|c|c|c|c|c|}
\hline & & solution & $\mathrm{E} / \mathrm{eV}$ & $\mathrm{E} / \mathrm{eV}$ & $\mathrm{E} / \mathrm{cm}^{-1}$ \\
\hline \multirow[t]{8}{*}{ optB86b-vdW } & $100 \mathrm{~K}$ & AFM & -513.4831 & -0.7124 & -5745 \\
\hline & & FM & -512.7707 & 0 & 0 \\
\hline & $300 \mathrm{~K}$ part1 & AFM & -513.0574 & -0.6112 & -4929 \\
\hline & & FM & -512.4462 & 0 & 0 \\
\hline & $300 \mathrm{~K}$ average & AFM & -513.0421 & -0.6841 & -5518 \\
\hline & & $\mathrm{FM}$ & -512.3580 & 0 & 0 \\
\hline & $300 \mathrm{~K}$ distorted & AFM & -513.0371 & -0.6407 & -5167 \\
\hline & & FM & -512.3964 & 0 & 0 \\
\hline \multirow[t]{4}{*}{ B3LYP } & $100 \mathrm{~K}$ & AFM & -719.6511 & -0.0619 & -499 \\
\hline & & FM & -719.5892 & 0 & 0 \\
\hline & $300 \mathrm{~K}$ part1 & AFM & -719.6589 & -0.2306 & -1860 \\
\hline & & FM & -719.4283 & 0 & 0 \\
\hline
\end{tabular}

${ }^{a}$ The B3LYP results correspond to single-point calculations on the basis of the optB86b-vdW optimized structures.

interactions, and strongly depends on the structure considered. For the actual distorted structure, these interactions are only slightly affected, in line with the small modifications of the $\mathrm{CuCl}_{4}^{2-}$ units. However, the supposed square-planar coordination (300 $\mathrm{K}$-average) would result in a significant enhancement of the organic-inorganic interactions, by almost 1 order of magnitude, reaching values in clear competition with the interdimer interaction (Table 5). A square-planar coordination would favor a larger contribution of the $\mathrm{Cl} 3 \mathrm{p}$ orbitals on the magnetic orbitals localized on the $\mathrm{CuCl}_{4}{ }^{2-}$ ions (Figure 8). The change in the relative orientation of the $\mathrm{CuCl}_{4}{ }^{2-}$ ions with respect to the organic units also affects the overlap between the organic and inorganic components, much more efficient for the case of an alternative square-planar geometry. Both factors favor the antiferromagnetic contribution of the magnetic coupling interaction, resulting in an enhancement of this interaction.

4.6. Periodic Plane Waves Calculations. We have performed periodic plane waves calculations with a 3-fold aim: (i) to have a global picture of the system taking into account all the long-range ion-ion interactions, (ii) to validate the CI evaluations of the dominant magnetic couplings, and (iii) to obtain the density of states (DOS) of the salt. Both the atomic positions and lattice parameters are optimized for two solutions; the results are shown in Tables 6 and 7 and S4 and S5. For the $300 \mathrm{~K}$ structure, three different sets of coordinates have been employed, part1 and average, with square-planar coordination for the $\mathrm{CuCl}_{4}{ }^{2-}$ ions, with the main difference 

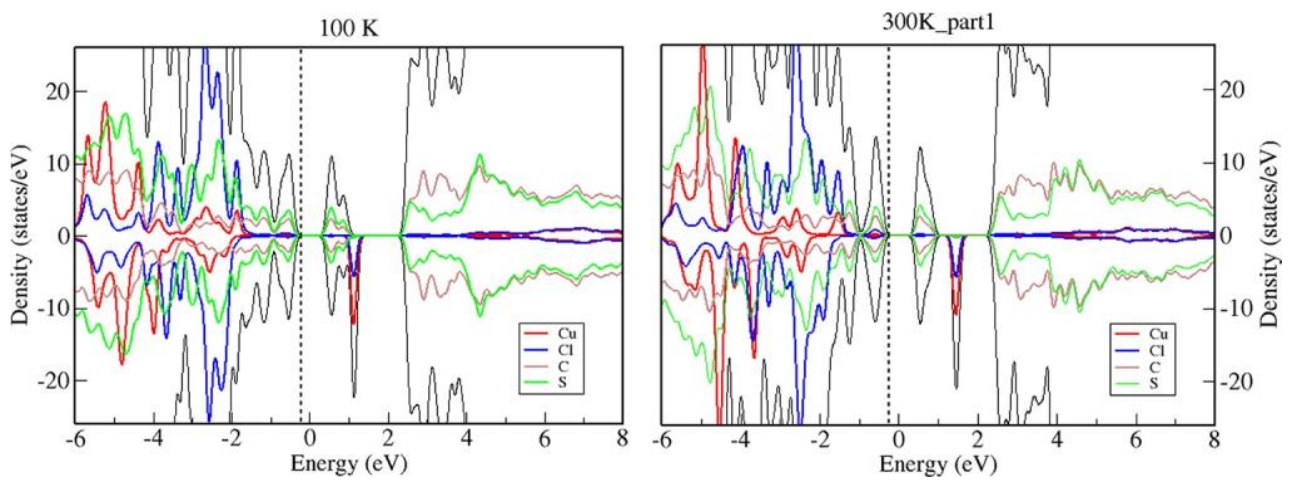

Figure 9. Density of states (black line) for the lowest energy AFM magnetic solution at $100 \mathrm{~K}$ (left) and $300 \mathrm{~K}$ (right), projected on the Cu (red), $\mathrm{Cl}$ (blue), C (brown), and S (green) atoms. The vertical dotted line represents the Fermi level.

among these three sets of coordinates concerning the relative position of the $\mathrm{Cl}$ atoms with respect to the organic molecule, and the distorted set, where the $\mathrm{CuCl}_{4}{ }^{2-}$ ions exhibit the actual distorted pseudo-4-fold symmetry, in between square planar and tetrahedral. The optimizations for the ground state AFM solution provide lattice and packing parameters in good agreement with the experimental data (Tables 6 and 7). The disagreements found in Table 7 for the optimized $300 \mathrm{~K}$ distorted cell are due to the presence of the pseudocenter of symmetry at the $\mathrm{Cu}$ position that cannot be modeled in the periodic calculations. In order to deal with this inconsistency, we used a double cell containing two $\mathrm{CuCl}_{4}{ }^{2-}$ ions, each adopting one of the two equally probable distorted orientations (Figure S3).

At both temperatures, the most stable solution corresponds to the AFM one, where the six unpaired electrons per cell are arranged in such way that four of them are paired and located on the organic radical and the other two remain unpaired on the $\mathrm{CuCl}_{4}{ }^{2-}$ ions. The completely ferromagnetic solution (NUPDOWN $=6)$ is also calculated to obtain an estimate of the amplitude of the magnetic interactions in the crystal and compare with the evaluations on the cluster fragments.

The changes observed as temperature is increased are well reproduced by the periodic calculations, indicating that the choices made during the optimizations (functionals and corrections, number of plane waves, kspacing, convergence criteria, ...) are adequate for this system. The most remarkable change induced by temperature is the lengthening of the closest $S-S$ contact (Table 7), related to the attenuation of the amplitude of the magnetic interactions.

Regarding the energies, the difference between the AFM and FM solutions gives an estimate of the dominant magnetic interactions in the organic stacks together with the contribution of the organic-inorganic couplings: $E(A F M)-E(F M)=J+$ $J_{\text {inter }}+J_{\mathrm{CuA}}+J_{\mathrm{CuB}}$ (Table 8). The amplitudes of these interactions are in agreement with those resulting from the DDCI calculations. Then, two different approaches (DFT and CI calculations) on different computational models (periodic and finite cluster models) provide the same picture for this system: the BEDT-TTF ${ }^{+}$radicals form strongly antiferromagnetic coupled dimers, $\mathrm{AB}$, that interact through antiferromagnetic interactions along the organic stacks. The periodic calculations also show the reduction of the AFM-FM separation when temperature increases (Table 8), in line with the attenuation of the antiferromagnetic couplings among the radical BEDT-TTF ${ }^{+}$units.
Finally, we have also calculated the density of states for the AFM solution at both temperatures. Figure 9 shows the electronic density of states for the magnetic solutions in the case of the AFM solution, just for the optimized structures at 100 and $300 \mathrm{~K}$. In both cases, using the optB86b-vdW functional, the semiconductor nature of the system cannot be reproduced. Instead, the DOS were obtained from single-point calculations with the B3LYP ${ }^{25}$ hybrid functional, known as an adequate option to predict the gap in material design. ${ }^{34}$ An examination of DOS curves makes clear the semiconductor character of the system for both 100 and $300 \mathrm{~K}$. The projected DOS on the different atoms illustrates the origin of the band edges in both cases. It is clear that both the conduction and valence band edges are mainly contributed by the organic component of the crystal (brown and green curves). On the other hand, the projected DOS on $\mathrm{Cu}$ and $\mathrm{Cl}$ (Figure 9) shows that the unpaired electrons in the AFM solution would be associated with the inorganic $\mathrm{CuCl}_{4}{ }^{2-}$ component.

4.7. Dynamic Thermal Effects. It is important to notice that all our calculations introduce the thermal effects in a static way, i.e., when temperature rises the structure changes, and our calculations are based on the X-ray crystallographic data recorded at a fixed temperature. However, it is also possible to conceive the presence of dynamic thermal effects that change instantaneously the structure of the system, in such a way that the X-ray structure just represents an average of all the different situations at a certain temperature.

In order to analyze the potential effect of the dynamic thermal agitation on the electronic structure of the system, we have carried out a computational experiment, similar to that performed by Robert et al. ${ }^{35,36}$ in verdazyl-based compounds. We have modified the relative slippage between the two BEDTTTF units by an horizontal movement that increases (decreases) the relative slippage of the AB (BA) dimer, maintaining fixed the intermolecular distance (Table S7). The total energies of the singlet and triplet states for both dimers are evaluated separately at the $\operatorname{CASSCF}(2 / 2)$ level, and the results are reported in Figure 10 and Table S7. The calculations take into account neither the effect of the environment nor the dynamical correlation but give an idea of the impact of small changes on the relative slippage.

For $\mathrm{AB}$ dimer, the increase of the slippage relieves part of the $\pi-\pi$ repulsions between the $S$ lone pairs and the central $\mathrm{C}-\mathrm{C}$ bond of the two units. The intradimer overlap is weakened, and the energy difference between the bonding and antibonding combinations of the SOMOs is reduced. Consequently, the $J$ coupling is significantly reduced. For BA dimer, the opposite 


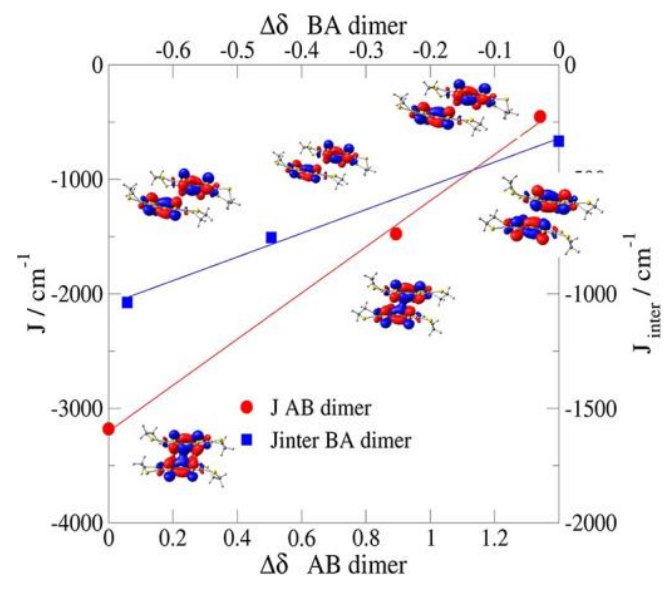

Figure 10. Effect of the change of slippage $\Delta \delta(\AA)$ of the BEDT-TTF units on the intradimer $J$ and interdimer $J_{\text {inter }}$ magnetic coupling constants. Evolution of the magnetic orbitals of the $\mathrm{AB}$ (bottom) and BA (top) dimers with changes in the slippage.

effect is observed. The slippage is reduced, the repulsions are enhanced, and the total energy increases for both states. The overlap between the SOMOs increases, and consequently the antiferromagnetic contribution to $J_{\text {inter }}$ is also enhanced. The $J_{\text {inter }}$ coupling value is noticeably augmented. This computational experiment, despite its simplicity, puts in evidence the potential and significant effect of the thermal fluctuations of the BEDT-TTF stacks on the magnetic properties of this system, as those observed in TTTA radicals by Vela et al. ${ }^{37}$ A correct evaluation of this effect would require the use of ab initio molecular dynamics (AIMD) approaches that could be particularly demanding on this complex system and clearly beyond the scope of this work.

\section{DISCUSSION AND COMPARISON WITH THE EXPERIMENTAL DATA}

The DDCI and periodic calculations on compound 1 have put in evidence the presence of a set of competing magnetic interactions, with different relative values depending on temperature. In both limit situations, the picture provided by the DDCI calculations is rather different from the simplified snapshot based on isolated BEDT-TTF dimers and noninteracting $\mathrm{CuCl}_{4}{ }^{2-}$ ions. The intradimer interaction is strong, but dimers are not isolated. They interact among them, resulting in alternating antiferromagnetic BEDT-TTF chains, as shown in Figure 11a. Additionally, the BEDT-TTF chains are not isolated, they interact with each other through the $\mathrm{CuCl}_{4}{ }^{2-}$ ions. The correct magnetic model corresponds to a spin-ladder, as shown in Figure 11b. The legs contain the BEDT-TTF stacks, where two antiferromagnetic interactions $\mathrm{J}$ and $\mathrm{J}_{\text {inter }}$ alternate (bold and single lines in Figure 11, respectively). The rungs take into account the organic-inorganic interactions, $\mathrm{A}-\mathrm{Cu}$ and $\mathrm{B}-\mathrm{Cu}$ (red lines in Figure 11). Additionally, there exists second-neighbor interactions among the $\mathrm{CuCl}_{4}{ }^{2-}$ ions and the organic molecules, corresponding to $\mathrm{J}_{\mathrm{A}-\mathrm{Cu}^{\prime}}$ and $\mathrm{J}_{\mathrm{B}-\mathrm{Cu}^{\prime}}$ in Table 6, represented with dashed lines in Figure $11 \mathrm{c}$.

We have carried out different attempts to simulate the $X^{T}$ curve. Our strategy consists in the definition of a spin model based on the amplitude of the different calculated interactions, and the diagonalization of the resulting Heisenberg Hamiltonian. The energy levels resulting from the diagonalization are substituted in the equation of $X(T)$ derived from statistical mechanics. The diagonalization and calculation of $X(\mathrm{~T})$ data are performed with $\mathrm{PHI}^{38}$ and MAGPACK ${ }^{39}$ packages. Taking into account the constraints of these packages (spin models containing up to 14 sites) and the stoichiometry of the crystal, all the spin models considered are restrained to $\mathrm{N}=12$ sites.

First, we have studied a $1 \mathrm{D}$ alternating $\mathrm{AF}$ chain with eight organic molecules and four noninteracting $S=1 / 2$ sites, representing the $\mathrm{CuCl}_{4}{ }^{2-}$ ions (red line in Figure 12), and we have compared the

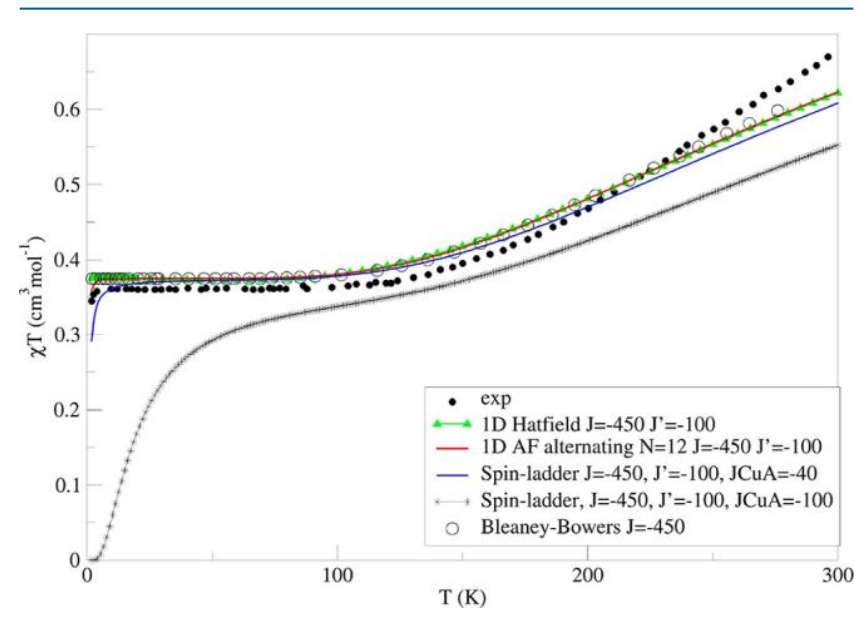

Figure 12. Simulation of the $\mathrm{X}^{\top}$ curve using different spin models. All the $\mathrm{J}$ values in $\mathrm{cm}^{-1}$.

resulting curve with that provided by the analytical expressions of Hatfield et al. $^{40}$ for the $1 \mathrm{D}$ alternating AF chain (closed green triangles). The agreement between both fittings confirms the absence of size-effects, at least for the 1D model. Additionally, we have used

$$
\begin{aligned}
& 1 \\
& 1 \\
& 1 \\
& 1 \\
& 1 \\
& \vdots \\
& \vdots \\
& \vdots
\end{aligned}
$$

(a)

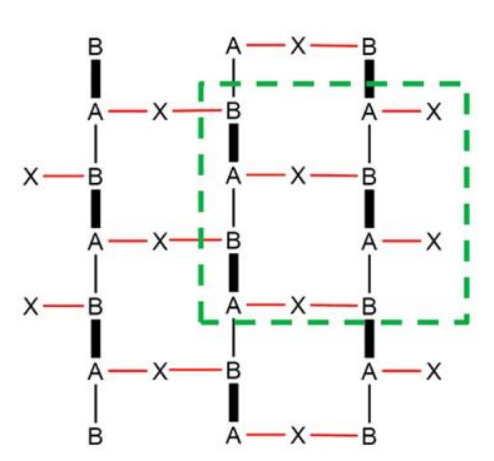

(b)

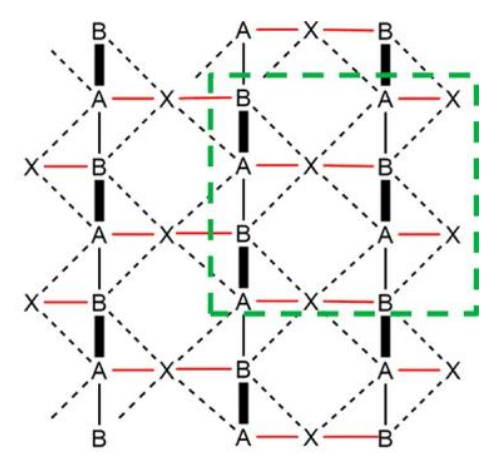

(c)

Figure 11. (a) 1D alternating antiferromagnetic BEDT-TTF chains, where the bold and single lines represent, respectively, the intradimer $J$ and interdimer $J_{\text {inter }}$ couplings, (b) 2D spin-ladders, that additionally include the $J_{\mathrm{ACu}}$ and $J_{\mathrm{BCu}}$ interactions (red lines), (c) 2D spin-ladder taking into account also the second-neighbor $\mathrm{J}_{\mathrm{A}-\mathrm{Cu}^{\prime}}$ and $\mathrm{J}_{\mathrm{B}-\mathrm{Cu}}$ interactions (dashed lines). The green box corresponds to the 12-site magnetic model employed in the simulations of the $\mathrm{X}^{\top}$ curve. $\mathrm{X}$ represents the $\mathrm{CuCl}_{4}{ }^{2-}$ ions. 
two spin-ladder models containing $\mathrm{N}=12$ sites (represented in Figure 12 with a green box), where eight sites correspond to the organic spins, and four sites represent the $\mathrm{CuCl}_{4}{ }^{2-}$ ions. Only the results of the model in Figure 11b are shown (blue line and stars curves). Similar behavior is obtained for the extended spin-ladder in Figure 11c. The three models present the same behavior, with the spin-ladder model being able to reproduce the drop of the $\mathrm{X}^{\top}$ curve at very low temperature in the case of a weak organic-inorganic coupling (blue line in Figure 12).

However, none of these three models provide a correct fitting when using the calculated $J$ values for the intradimer interaction. Figure 13

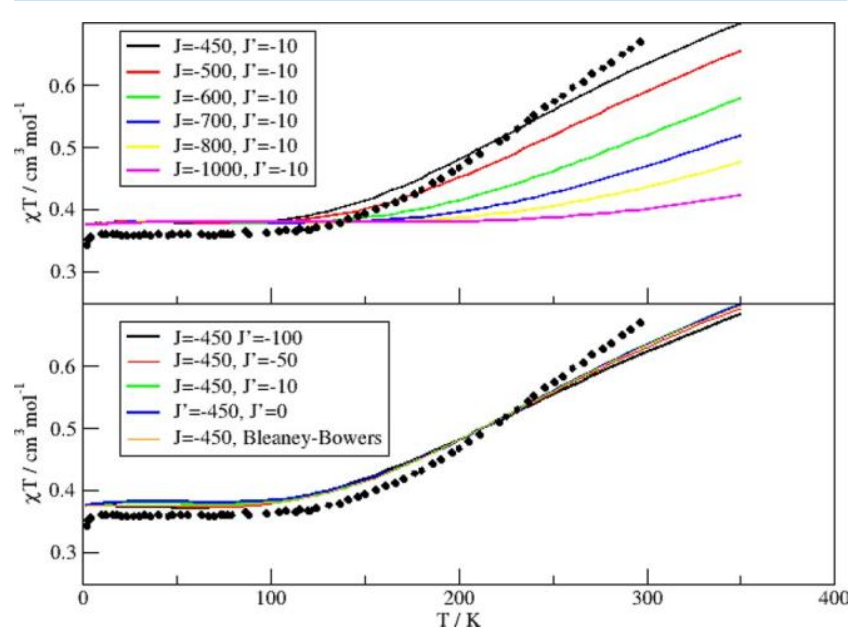

Figure 13. Plots of the $X^{\top}$ product provided by Hatfield's model with different sets of $J$ and $J^{\prime}$ values (in $\mathrm{cm}^{-1}$ ). The filled black circles correspond to the experimental values.

represents the $\mathrm{X}^{\top}$ product provided by the Hatfield model for the 1D chain with two alternating AF interactions, $J$ and $J^{\prime}$, plus the constant contribution of the isolated $S=1 / 2$ spin of the $\mathrm{CuCl}_{4}{ }^{2-}$ ion, for different values of $J$ and $J^{\prime}$ : the model is almost not sensitive to the $J^{\prime}$ value; it provides fittings of reasonable quality for very different values of J', but it shows a strong dependence on the amplitude of $J$. When $J$ increases, the quality of the fitting gets worse, for $J \sim-1000 \mathrm{~cm}^{-1}$ the $\mathrm{X}^{\top}$ product is constant up to $300 \mathrm{~K}$ since the only contribution to $\mathrm{X}^{\mathrm{T}}$ comes from the $\mathrm{CuCl}_{4}{ }^{2-}$ ions, here considered isolated. Again, the $X^{\top}$ curves using Hatfield's model with $\mathrm{J} \sim-1000 \mathrm{~cm}^{-1}$ and very different values of $J^{\prime}$ are quasi-indistinguishable. The same behavior is obtained when using the calculated $J$ and $J_{\text {intra }}$ values, $J$ is so large than the $x^{\top}$ curve is completely flat for all the temperature range. Then in all the models considered in Figure 12, it is necessary to use an attenuated value for the dominant $J$ coupling; otherwise, the resulting curves do not represent correctly the experimental behavior.

This requirement, that could at first glance put in question the reliability of the calculated $J$ values, could also be related to the reduced size of the magnetic model employed in the simulation, but also to an intrinsic limitation imposed by the relative amplitude of the main interactions in this compound. The absence of size-effects is confirmed at least for the $1 \mathrm{D}$ alternating model, since the same $\mathrm{XT}^{\mathrm{T}}$ curve is obtained using a 12-site model than the analytical expression of Hatfield for the infinite chain. Regarding the relative amplitude of the main interactions, the intradimer interaction is far larger than $k_{B} T$ over the working temperature and always larger than the interdimer interaction. This imposes an antiferromagnetic distribution inside the dimer for all the registered temperatures and implies that the observed thermal dependence of XT is mainly due to the interdimer interactions. Then the $J$ value supplied by the best fittings does not correspond to the intradimer coupling but to the interdimer interaction. For this reason, in all the models considered, the fitting is correct only when the dominant interaction reaches a value of about $-450 \mathrm{~cm}^{-1}$, close to the calculated interdimer coupling constant. And also for this reason the simplest of the considered models, the Bleaney-Bowers one (open circles in Figure 12), is able to reproduce the experimental curve, with only one effective parameter, assigned to the intradimer coupling in ref 7 but really representing the interdimer interactions.

An additional ingredient to be taken into account is the dynamic thermal effects discussed above. When temperature increases, the thermal fluctuations of the BEDT-TTF stacks could significantly modify both the intradimer and interdimer interactions, as occurs for other systems with $\pi$ stacking, ${ }^{35,36}$ resulting in $J$ and $J_{\text {intra }}$ values in a closer similarity than those arising from an static analysis of the interactions. This reinforces the idea of the effective nature of the interaction parameter provided by the fittings.

\section{CONCLUSIONS}

Multifunctional hybrid materials are investigated as potential candidates in many technological applications. They combine organic and inorganic components with different individual properties, with the aim of obtaining a single compound showing both properties simultaneously or new ones due to the competition and interplay of these properties. The interpretation and rationalization of the behavior of the resulting system are crucial for designing and providing new materials with optimized properties. Most of the interpretations are based on simplified models, and this could be a serious bias in the understanding of the phenomena at the molecular level.

This work has evidenced how the macroscopic behavior of an apparently simple hybrid salt $[\mathrm{BEDT}-\mathrm{TTF}]_{2}\left[\mathrm{CuCl}_{4}\right]$ can be explained with a simple model, which does not correlate with the local interactions present in the system. This model of noninteracting dimers of $\mathrm{BEDT}_{-} \mathrm{TTF}^{+}$and isolated $\mathrm{CuCl}_{4}{ }^{2-}$ ions contrasts with that extracted from the theoretical calculations: alternating antiferromagnetic 1D chains of the organic BEDT- $\mathrm{TTF}^{+}$radical, connected through weak antiferromagnetic interactions with the $\mathrm{CuCl}_{4}{ }^{2-}$ ions. The combination of extended CI and periodic plane waves calculations gives the clues of this apparent inconsistency and explains why the simplified model fits the data.

The theoretical calculations performed on this system are far from being routine jobs. To our knowledge this is the first theoretical study combining both extended CI and periodic DFT calculations on a system of such complexity: a system combining organic radicals with inorganic magnetic ions, very different in nature (itinerant vs localized spins), with very large unit cells, presenting through-space interactions mediated by hydrogen bonds and $\pi-\pi$ contacts, with interactions rather weak which require a precise and exigent evaluation of the energies of the spin states.

The study highlights the potential of these calculations on the understanding of the properties of these complex systems, and its conclusions could be relevant not only for the particular compound analyzed but for many other radical cation salts based on BEDT-TTF or related TTF radical cations.

\section{ASSOCIATED CONTENT}

\section{Supporting Information}

The Supporting Information is available free of charge on the ACS Publications website at DOI: 10.1021/acs.inorgchem.7b03240.

Computational details of the finite cluster and periodic calculations, effective Heisenberg Hamiltonians for different cluster models, valence-bond analysis of the $\mathrm{AB}$ and $\mathrm{BA}$ singlet ground state, effect of the change of slippage on the magnetic coupling constant and relative energies of $\mathrm{AB}$ and $\mathrm{BA}$ dimers, equilibrium cell 
parameters and molecular package parameters for AFM and $\mathrm{FM}$ solutions and active orbitals for $\mathrm{AB}+2 \mathrm{CuCl}_{4}$ and $\mathrm{BA}+2 \mathrm{CuCl}_{4}$ clusters at $300 \mathrm{~K}$. (PDF)

\section{Accession Codes}

CCDC 1590172 and 1831182-1831184 contain the supplementary crystallographic data for this paper. These data can be obtained free of charge via www.ccdc.cam.ac.uk/data_request/ cif, or by emailing data_request@ccdc.cam.ac.uk, or by contacting The Cambridge Crystallographic Data Centre, 12 Union Road, Cambridge CB2 1EZ, UK; fax: +44 1223336033.

\section{AUTHOR INFORMATION}

\section{Corresponding Author}

*E-mail: calzado@us.es.

\section{ORCID $\odot$}

Carmen J. Calzado: 0000-0003-3841-7330

José Ramón Galán Mascaros: 0000-0001-7983-9762

Notes

The authors declare no competing financial interest.

\section{ACKNOWLEDGMENTS}

The authors acknowledge Dr. Daniel Maynau for valuable comments. The authors acknowledge the financial support provided by the Ministerio de Economia y Competitividad (Spain) and FEDER funds through the projects CTQ201569019-P (MINECO/FEDER), CTQ2015-71287-R, and the Severo Ochoa Excellence Accreditation 2014-2018 SEV-20130319, the Generalitat de Catalunya (2014-SGR-797 and the CERCA program). The authors also thank the technical support of the Supercomputing Team of the Centro Informatico Cientifico de Andalucia (CICA) and of the X-ray Diffraction Unit at ICIQ.

\section{REFERENCES}

(1) (a) Ouahab, L. Coordination complexes in conducting and magnetic molecular materials. Coord. Chem. Rev. 1998, 178, 15011531. (b) Coronado, E.; Day, P. Magnetic molecular conductors. Chem. Rev. 2004, 104, 5419-5448. (c) Enoki, T.; Miyazaki, A. Magnetic TTF-based charge-transfer complexes. Chem. Rev. 2004, 104, $5449-5477$.

(2) (a) Kurmoo, M.; Graham, A. W.; Day, P.; Coles, S. J.; Hursthouse, M. B.; Caulfield, J. L.; Singleton, J.; Pratt, F. L.; Hayes, W.; Ducasse, L.; Guionneau, P. Superconducting and semiconducting magnetic charge-transfer salts- (BEDT-TTF $)_{4} \mathrm{AFe}\left(\mathrm{C}_{2} \mathrm{O}_{4}\right)_{3}-\mathrm{C}_{6} \mathrm{H}_{5} \mathrm{CN}$ $\left(\mathrm{A}=\mathrm{H}_{2} \mathrm{O}, \mathrm{K}, \mathrm{NH}_{4}\right)$. J. Am. Chem. Soc. 1995, 117, 12209-12217. (b) Martin, L.; Turner, S. S.; Day, P.; Guionneau, P.; Howard, J. A. K.; Hibbs, D. E.; Light, M. E.; Hursthouse, M. B.; Uruichi, M.; Yakushi, K. Crystal chemistry and physical properties of superconducting and semiconducting charge transfer salts of the type (BEDT-TTF) $\mathrm{A}(\mathrm{I}) \mathrm{M}(\mathrm{III})\left(\mathrm{C}_{2} \mathrm{O}_{4}\right)_{3} \mathrm{PhCN}\left(\mathrm{A}(\mathrm{I})=\mathrm{H}_{3} \mathrm{O}, \mathrm{NH}_{4}, \mathrm{~K} ; \mathrm{M}-\mathrm{III}=\mathrm{Cr}, \mathrm{Fe}\right.$, $\mathrm{Co}, \mathrm{Al}$; $\mathrm{BEDT}-\mathrm{TTF}=$ bis (ethylenedithio)tetrathiafulvalene). Inorg. Chem. 2001, 40, 1363-1371. (c) Kobayashi, H.; Kobayashi, A.; Cassoux, P. BETS as a source of molecular magnetic superconductors (BETS $=$ bis(ethylenedithio)tetraselenafulvalene). Chem. Soc. Rev. 2000, 29, 325-333. (d) Uji, S.; Shinagawa, H.; Terashima, T.; Yakabe, T.; Terai, Y.; Tokumoto, M.; Kobayashi, A.; Tanaka, H.; Kobayashi, H. Magnetic-field-induced superconductivity in a two-dimensional organic conductor. Nature 2001, 410, 908-910.

(3) (a) Coronado, E.; Galan-Mascaros, J. R.; Gomez-Garcia, C. J.; Laukhin, V. Coexistence of ferromagnetism and metallic conductivity in a molecule-based layered compound. Nature 2000, 408, 447-449. (b) Galan-Mascaros, J. R.; Coronado, E. Molecule-based ferromagnetic conductors: Strategy and design. C. R. Chim. 2008, 11, 1110-1116. (c) Coronado, E.; Galan-Mascaros, J. R.; Gomez-Garcia, C. J.;
Martinez-Ferrero, E.; van Smaalen, S. Incommensurate nature of the multilayered molecular ferromagnetic metals based on bis(ethylenedithio)tetrathiafulvalene and bimetallic oxalate complexes. Inorg. Chem. 2004, 43, 4808-4810. (d) Alberola, A.; Coronado, E.; Galan-Mascaros, J. R.; Gimenez-Saiz, C.; Gomez-Garcia, C. J. A molecular metal ferromagnet from the organic donor bis(ethylenedithio)tetraselenafulvalene and bimetallic oxalate complexes. J. Am. Chem. Soc. 2003, 125, 10774-10775.

(4) (a) Coronado, E.; Galan-Mascaros, J. R.; Gomez-Garcia, C. J.; Murcia-Martinez, A.; Canadell, E. A chiral molecular conductor: Synthesis, structure, and physical properties of ET (3) Sb-2(L-tart)(2). $\mathrm{CH}_{3} \mathrm{CN}$ (ET = Bis(ethylendithio)tetrathiafulvalene; L-tart $=(2 \mathrm{R}, 3 \mathrm{R})-$ (+)-tartrate). Inorg. Chem. 2004, 43, 8072-8077. (b) Riobe, F.; Piron, F.; Rethore, C.; Madalan, A. M.; Gomez-Garcia, C. J.; Lacour, J.; Wallis, J. D.; Avarvari, N. Radical cation salts of BEDT-TTF, enantiopure tetramethyl-BEDT-TTF, and TTF-Oxazoline (TTF-Ox) donors with the homoleptic TRISPHAT anion. New J. Chem. 2011, 35, 2279-2286. (c) Brezgunova, M.; Shin, K.-S.; Auban-Senzier, P.; Jeannin, O.; Fourmigue, M. Combining halogen bonding and chirality in a two-dimensional organic metal (EDT-TTF- $\left.\mathrm{I}_{2}\right)_{2}$ (D-camphorsulfonate) $\mathrm{H}_{2} \mathrm{O}$. Chem. Commun. 2010, 46, 3926-3928. (d) Martin, L.; Day, P.; Horton, P.; Nakatsuji, S. i.; Yamada, J. i.; Akutsu, H. Chiral conducting salts of BEDT-TTF containing a single enantiomer of tris(oxalato)chromate(III) crystallised from a chiral solvent. J. Mater. Chem. 2010, 20, 2738-2742. (e) Chmel, N. P.; Allan, L. E. N.; Becker, J. M.; Clarkson, G. J.; Turner, S. S.; Scott, P. TTF salts of optically pure cobalt pyridine amidates; detection of soluble assemblies with stoichiometry corresponding to the solid state. Dalton Trans. 2011, 40, 1722-1731. (f) Wallis, J. D.; Griffiths, J. P. Substituted BEDT-TTF derivatives: synthesis, chirality, properties potential applications. J. Mater. Chem. 2005, 15, 347-365.

(5) Coronado, E.; Marti-Gastaldo, C.; Navarro-Moratalla, E.; Ribera, A.; Galan-Mascaros, J. R. Intercalation of two-dimensional oxalatebridged molecule-based magnets into layered double hydroxide hosts.

J. Mater. Chem. 2010, 20, 9476-9483.

(6) Benmansour, S.; Sanchez-Maez, Y.; Gomez-García, C. MnContaining Paramagnetic Conductors with Bis(ethylenedithio)tetrathiafulvalene (BEDT-TTF). Magnetochemistry 2017, 3, 7.

(7) Rodríguez-García, B.; Goberna-Ferron, S.; Koo, Y.-S.; BenetBuchholz, J.; Galan-Mascaros, J. R. Nonelectrochemical Synthesis, Crystal Structure, and Physical Properties of the Radical Salt [ET]2[CuCl4] $(\mathrm{ET}=$ Bis(ethylenedithio)tetrathiafulvalene $)$. Inorg. Chem. 2013, 52, 14376-14381.

(8) Mori, T.; Sakai, F.; Saito, G.; Inokuchi, H. Structural and Electrical Properties of (BEDT-TTF) ${ }_{3} \mathrm{CuBr}_{3}$. Chem. Lett. 1987, 16, 927-930.

(9) Mallah, T.; Hollis, C.; Bott, S.; Kurmoo, M.; Day, P.; Allan, M.; Friend, R. H. Crystal structures and physical properties of bis(ethylenedithio)-tetrathiafulvalene charge-transfer salts with $\mathrm{FeX}_{4}$ (X $=\mathrm{Cl}$ or Br) anions. J. Chem. Soc., Dalton Trans. 1990, 859-865.

(10) Korotkov, V. E.; Shibaeva, R. P. Kristallografiya (Russ.) (Crystallogr. Rep.) 1989, 34, 1442.

(11) Geiser, U.; Anderson, B. A.; Murray, A.; Pipan, C. M.; Rohl, C. A.; Vogt, B. A.; Wang, H. H.; Williams, J. M.; Kang, D. B.; Whangbo, M.-H. BEDT-TTF Salts with Square-Planar Gold(III) Complex Anions: $\beta$-(ET) ${ }_{2} \mathrm{AuCl}_{4}$ and $(\mathrm{ET})_{2} \mathrm{Au}(\mathrm{CN})_{2} \mathrm{Cl}_{2}$. Mol. Cryst. Liq. Cryst. 1990, 181, 105-116.

(12) Kahn, O. Molecular magnetism; VCH: New York, NY, 1993; p xvi, $380 \mathrm{p}$.

(13) (a) Miralles, J.; Castell, O.; Caballol, R.; Malrieu, J.-P. Specific CI calculation of energy differences: Transition energies and bond energies. Chem. Phys. 1993, 172, 33-43. (b) Miralles, J.; Daudey, J.-P.; Caballol, R. Variational calculation of small energy differences. The singlet-triplet gap in $\left[\mathrm{Cu}_{2} \mathrm{Cl}_{6}\right]^{2-}$. Chem. Phys. Lett. 1992, 198, 555562 .

(14) Bories, B.; Maynau, D.; Bonnet, M.-L. Selected excitation for CAS-SDCI calculations. J. Comput. Chem. 2007, 28, 632-643.

(15) Tenti, L.; Maynau, D.; Angeli, C.; Calzado, C. J. Highly efficient perturbative plus variational strategy based on orthogonal valence 
bond theory for the evaluation of magnetic coupling constants. Application to the trinuclear $\mathrm{Cu}$ (II) site of multicopper oxidases. Phys. Chem. Chem. Phys. 2016, 18, 18365-18380.

(16) Calzado, C. J. On the Controversial Fitting of Susceptibility Curves of Ferromagnetic CuII Cubanes: Insights from Theoretical Calculations. Chem. - Eur. J. 2013, 19, 1254-1261.

(17) Calzado, C. J.; Ben Amor, N.; Maynau, D. Magnetic Coupling Constants of Self-Assembled Cu-II [3 3 3] Grids: Alternative Spin Model from Theoretical Calculations. Chem. - Eur. J. 2014, 20, 89798987.

(18) Chang, C.; Calzado, C. J.; Ben Amor, N.; Sanchez Marin, J.; Maynau, D. Multi-scale multireference configuration interaction calculations for large systems using localized orbitals: Partition in zones. J. Chem. Phys. 2012, 137, 104102.

(19) Jung, J.; Puget, M.; Cador, O.; Bernot, K.; Calzado, C. J.; Le Guennic, B. Analysis of the Magnetic Exchange Interactions in Yttrium(III) Complexes Containing Nitronyl Nitroxide Radicals. Inorg. Chem. 2017, 56, 6788-6801.

(20) Aquilante, F.; De Vico, L.; Ferre, N.; Ghigo, G.; Malmqvist, P.A.; Neogrady, P.; Pedersen, T. B.; Pitonak, M.; Reiher, M.; Roos, B. O.; Serrano-Andres, L.; Urban, M.; Veryazov, V.; Lindh, R. Software News and Update MOLCAS 7: The Next Generation. J. Comput. Chem. 2010, 31, 224-247.

(21) Maynau, D. CASDI package developed at the Laboratoire de Physique Quantique; Université Paul Sabatier, Toulouse, France, 1999. (22) Ben Amor, N.; Maynau, D. Size-consistent self-consistent configuration interaction from a complete active space. Chem. Phys. Lett. 1998, 286, 211-220.

(23) (a) Kresse, G.; Furthmuller, J. Efficient iterative schemes for ab initio total-energy calculations using a plane-wave basis set. Phys. Rev. B: Condens. Matter Mater. Phys. 1996, 54, 11169-11186. (b) Kresse, G.; Furthmuller, J. Efficiency of ab-initio total energy calculations for metals and semiconductors using a plane-wave basis set. Comput. Mater. Sci. 1996, 6, 15-50. (c) Kresse, G.; Hafner, J. Ab-initio molecular-dynamics simulation of the liquid-metal amorphous-semiconductor transition in germanium. Phys. Rev. B: Condens. Matter Mater. Phys. 1994, 49, 14251-14269. (d) Kresse, G.; Hafner, J. Ab initio molecular-dynamics for liquid-metals. Phys. Rev. B: Condens. Matter Mater. Phys. 1993, 47, 558-561. (e) Kresse, G.; Joubert, D. From ultrasoft pseudopotentials to the projector augmented-wave method. Phys. Rev. B: Condens. Matter Mater. Phys. 1999, 59, 17581775. (f) Blochl, P. E. Projector augmented-wave method. Phys. Rev. B: Condens. Matter Mater. Phys. 1994, 50, 17953-17979.

(24) (a) Klimes, J.; Bowler, D. R.; Michaelides, A. Chemical accuracy for the van der Waals density functional. J. Phys.: Condens. Matter 2010, 22, 022201. (b) Klimes, J.; Bowler, D. R.; Michaelides, A. Van der Waals density functionals applied to solids. Phys. Rev. B: Condens. Matter Mater. Phys. 2011, 83, 195131.

(25) (a) Stephens, P. J.; Devlin, F. J.; Chabalowski, C. F.; Frisch, M. J. $\mathrm{Ab}$ initio calculation of vibrational absorption and circular-dichroism spectra using density-functional force-fields. J. Phys. Chem. 1994, 98, 11623-11627. (b) Becke, A. D. Density-functional thermochemistry. 3. The role of exact exchange. J. Chem. Phys. 1993, 98, 5648-5652.

(26) (a) Miller, J. S. Four-Center Carbon-Carbon Bonding. Acc. Chem. Res. 2007, 40, 189-196. (b) Casado, J.; Burrezo, P. M.; Ramírez, F. J.; Navarrete, J. T. L.; Lapidus, S. H.; Stephens, P. W.; Vo, H.-L.; Miller, J. S.; Mota, F.; Novoa, J. J. Evidence for Multicenter Bonding in Dianionic Tetracyanoethylene Dimers by Raman Spectroscopy. Angew. Chem., Int. Ed. 2013, 52, 6421-6425. (c) Del Sesto, R. E.; Miller, J. S.; Lafuente, P.; Novoa, J. J. Exceptionally Long $(\geq 2.9$ $2-$

A) CC Bonding Interactions in $\pi-[\mathrm{TCNE}]_{2}$ Dimers: Two-Electron Four-Center Cation-Mediated CC Bonding Interactions Involving $\pi^{*}$ Electrons. Chem. - Eur. J. 2002, 8, 4894-4908.

(27) García-Yoldi, I.; Mota, F.; Novoa, J. J. The origin of the twoelectron/four-centers $\mathrm{C}-\mathrm{C}$ bond in $\pi-\mathrm{TCNE}_{2}{ }^{2-}$ dimers: Electrostatic or dispersion? J. Comput. Chem. 2007, 28, 326-334.

(28) Domingo, A.; Verot, M.; Mota, F.; de Graaf, C.; Novoa, J. J.; Robert, V. Impact of short and long-range effects on the magnetic interactions in neutral organic radical-based materials. Phys. Chem. Chem. Phys. 2013, 15, 6982-6989.

(29) Domingo, A.; Rodríguez-Fortea, A.; Swart, M.; de Graaf, C.; Broer, R. Ab initio absorption spectrum of $\mathrm{NiO}$ combining molecular dynamics with the embedded cluster approach in a discrete reaction field. Phys. Rev. B: Condens. Matter Mater. Phys. 2012, 85, 155143.

(30) Zapata-Rivera, J.; Maynau, D.; Calzado, C. J. Evaluation of the Magnetic Interactions in Salts Containing $\left[\mathrm{Ni}(\mathrm{dmit})_{2}\right]^{-}$Radical Anions. Chem. Mater. 2017, 29, 4317-4329.

(31) (a) Mori, T.; Katsuhara, M.; Akutsu, H.; Kikuchi, K.; Yamada, J.; Fujiwara, H.; Matsumoto, T.; Sugimoto, T. Estimation of pdinteractions in magnetic molecular conductors. Polyhedron 2005, 24,

2315-2320. (b) Mori, T.; Katsuhara, M. Estimation of pd-interactions in organic conductors including magnetic anions. J. Phys. Soc. Jpn. 2002, 71, 826-844.

(32) Cui, H. B.; Kobayashi, H.; Kobayashi, A. Phase diagram and anomalous constant resistivity state of a magnetic organic superconducting alloy, $[\lambda]$-(BETS $)_{2} \mathrm{Fe}_{\mathrm{x}} \mathrm{Ga}_{1-\mathrm{x}} \mathrm{Cl}_{4}$. J. Mater. Chem. 2007, 17, 45.

(33) Murakawa, H.; Kanda, A.; Ikeda, M.; Matsuda, M.; Hanasaki, N. Giant ferromagnetic p-d interaction in a phthalocyanine molecule. Phys. Rev. B: Condens. Matter Mater. Phys. 2015, 92, 054429.

(34) Garza, A. J.; Scuseria, G. E, Predicting Band Gaps with Hybrid Density Functionals. J. Phys. Chem. Lett. 2016, 7, 4165-4170.

(35) Verot, M.; Brefuel, N.; Pecaut, J.; Train, C.; Robert, V. Seeking Hidden Magnetic Phenomena by Theoretical Means in a Thiooxoverdazyl Adduct. Chem. - Asian J. 2012, 7, 380-386.

(36) Rota, J.-B.; Le Guennic, B.; Robert, V. Toward Verdazyl RadicalBased Materials: Ab Initio Inspection of Potential Organic Candidates for Spin-Crossover Phenomenon. Inorg. Chem. 2010, 49, 1230-1237. (37) Vela, S.; Mota, F.; Deumal, M.; Suizu, R.; Shuku, Y.; Mizuno, A.; Awaga, K.; Shiga, M.; Novoa, J. J.; Ribas-Arino, J. The key role of vibrational entropy in the phase transitions of dithiazolyl-based bistable magnetic materials. Nat. Commun. 2014, 5, 4411.

(38) Chilton, N. F.; Anderson, R. P.; Turner, L. D.; Soncini, A.; Murray, K. S. PHI: A powerful new program for the analysis of anisotropic monomeric and exchange-coupled polynuclear d- and f-

block complexes. J. Comput. Chem. 2013, 34, 1164-1175.

(39) Borras-Almenar, J. J.; Clemente-Juan, J. M.; Coronado, E.; Tsukerblat, B. S. MAGPACK A package to calculate the energy levels, bulk magnetic properties, and inelastic neutron scattering spectra of high nuclearity spin clusters. J. Comput. Chem. 2001, 22, 985-991. (40) Hall, J. W.; Marsh, W. E.; Weller, R. R.; Hatfield, W. E. Exchange coupling in the alternating-chain compounds catena-di-.mu.chloro-bis(4-methylpyridine)copper(II), catena-di-.mu.-bromobis(Nmethylimidazole)copper(II), catena-[hexanedione)bis(thiosemicarbazonato)]copper(II), and catena-[octanedione bis (thiosemicarbazonato)]copper(II). Inorg. Chem. 1981, 20, 1033-1037. 